OPEN ACCESS

Edited by:

Wei Cao,

Shanghai Jiao Tong University, China

Reviewed by:

Yanjie Zhang,

Shanghai Jiao Tong University, China Xuan Zhou,

Tianjin Medical University Cancer

Institute and Hospital, China

*Correspondence:

Zhe Shao

shaozhe@whu.edu.cn

Zhengjun Shang

shangzhengjun@whu.edu.cn

Specialty section:

This article was submitted to Head and Neck Cancer,

a section of the journal

Frontiers in Oncology

Received: 13 October 2020 Accepted: 26 November 2020 Published: 15 January 2021

Citation:

$X u Y$, Jiang E, Shao Z and Shang $Z$ (2021) Long Noncoding RNAs

in the Metastasis of Oral

Squamous Cell Carcinoma.

Front. Oncol. 10:616717.

doi: 10.3389/fonc.2020.616717

\section{Long Noncoding RNAs in the Metastasis of Oral Squamous Cell Carcinoma}

\author{
Yuming Xu, Erhui Jiang, Zhe Shao* and Zhengjun Shang * \\ The State Key Laboratory Breeding Base of Basic Science of Stomatology (Hubei-MOST) \& Key Laboratory of Oral \\ Biomedicine Ministry of Education, School \& Hospital of Stomatology, Wuhan University, Wuhan, China
}

Oral squamous cell carcinoma (OSCC) is a common malignant tumor worldwide. Metastasis is the main cause of the death of OSCC patients. Long noncoding RNAs (IncRNAs), one of the key factors affecting OSCC metastasis, are a subtype of RNA with a length of more than 200 nucleotides that has little or no coding potential. In recent years, the important role played by IncRNAs in biological processes, such as chromatin modification, transcription regulation, RNA stability regulation, and mRNA translation, has been gradually revealed. More and more studies have shown that IncRNAs can regulate the metastasis of various tumors including OSCC at epigenetic, transcriptional, and post-transcriptional levels. In this review, we mainly discussed the role and possible mechanisms of IncRNAs in OSCC metastasis. Most IncRNAs act as oncogenes and only a few IncRNAs have been shown to inhibit OSCC metastasis. Besides, we briefly introduced the research status of cancer-associated fibroblasts-related IncRNAs in OSCC metastasis. Finally, we discussed the research prospects of IncRNAs-mediated crosstalk between OSCC cells and the tumor microenvironment in OSCC metastasis, especially the potential research value of exosomes and lymphangiogenesis. In general, IncRNAs are expected to be used for screening, treatment, and prognosis monitoring of OSCC metastasis, but more work is still required to better understand the biological function of IncRNAs.

Keywords: IncRNAs, cancer-associated fibroblasts, exosomes, oral squamous cell carcinoma, tumor microenvironment, metastasis

\section{INTRODUCTION}

Oral squamous cell carcinoma (OSCC) is the most common type of head and neck squamous cell carcinoma (HNSCC) which ranks sixth among cancers worldwide (1). The initiation and progression of OSCC are the results of the combined action of various factors, and tobacco, alcohol, and human papillomavirus (HPV) infection are considered as the main causes of OSCC (2). Surgical resection is an effective method to treat OSCC, and the surgical cure rate of patients with early-stage OSCC can reach more than $80 \%$ (3). However, the overall prognosis of patients with OSCC is poor, with a 5-year survival rate of only about $60 \%(4,5)$. Metastasis is the major factor leading to the low survival rate of OSCC patients, and the 5-year survival rate of OSCC patients with metastasis is only about $40 \%$ (5). Notably, 
about $40 \%$ of OSCC patients may have metastasis (5). In addition, for non-metastatic patients, local resection of the primary tumor can achieve an ideal therapeutic effect, while metastatic patients generally need neck lymph node dissection, which not only increases the risk of surgery but also often causes postoperative dysfunction and maxillofacial deformities $(6,7)$. Whereas, it is still difficult to accurately determine whether OSCC patients have metastasis before surgery, which may lead to over-medical treatment (performing neck dissection for patients without lymph node metastasis) or insufficient treatment (patients with lymph node metastasis did not undergo neck dissection). Therefore, finding effective methods to screen, block, and treat OSCC metastasis is a top priority in OSCC research.

The metastasis of OSCC has two characteristics $(5,8)$ : on the one hand, the metastasis rate of OSCC occurring in diverse anatomical parts is different, which is about $40 \%$ in the buccal and soft palate, but less than $30 \%$ in the tongue and floor of mouth; on the other hand, OSCC metastasis mainly manifests as regional cervical lymph node metastasis, distant metastasis is rare. Although the mechanisms behind this phenomenon are still unclear, current research indicated that enhanced migration and invasion ability of tumor cells (9) and lymphangiogenesis (10) may be critical factors that promote OSCC metastasis. Todorovic et al. found that the expression levels of plakoglobin and desmoglein 2 (both are involved in maintaining intercellular adhesion) in squamous carcinoma cells derived from the top of the oral cavity are significantly lower than those in squamous carcinoma cells derived from the tongue, which is helpful for the migration and invasion of cells, and may be used to explain why the metastasis rate of OSCC in the soft palate is higher than that in the tongue (9). By analyzing the lymphatic vessel density in paraffin-embedded OSCC tissues, Sasahira et al. discovered that high lymphatic vessel density is closely related to lymph node metastasis and poor prognosis, which suggests that lymphangiogenesis may play a key role in the metastasis of OSCC (10). Unfortunately, scholars still have a limited understanding of the mechanisms of OSCC migration, invasion, and lymphangiogenesis, and have yet to find effective biomarkers.

Long noncoding RNAs (lncRNAs) are a subtype of RNA with a length of more than 200 nucleotides that has little or no coding potential (11). There are large amounts of lncRNAs in human cells that $68 \%$ of the human genome transcripts can be classified as lncRNAs (12). The expression of lncRNAs has certain tissue specificity (13), which suggests that they may play an important role in the biological processes of cells. Studies have confirmed that lncRNAs can interact with biological macromolecules including chromatin, protein, and RNA (14). In addition, many lncRNAs have been found to be abnormally expressed in various cancers, such as liver cancer (15), breast cancer (16), bladder cancer (17), and colorectal cancer (18), and regulate tumor cell metastasis through different mechanisms. In studies related to OSCC, lncRNAs were elucidated to be involved in tumor initiation (19) and prognosis (20). Furthermore, lncRNAs, such as MALAT1 (metastasis associated lung adenocarcinoma transcript 1) (21), UCA1 (urothelial cancer-associated 1) (22), AFAP1-AS1 (actin filament associated protein 1 antisense RNA 1) (23) and MEG3 (maternally expressed gene 3) (24) have been shown to participate in the regulation of OSCC migration, invasion, and metastasis. These studies have demonstrated the potential of lncRNAs for screening, treatment, and prognostic monitoring of OSCC metastasis. Therefore, in this paper, we review the role and possible mechanisms of lncRNAs in OSCC metastasis. In particular, we introduced the research status of cancer-associated fibroblasts-related lncRNAs in OSCC metastasis and discussed the research prospects of lncRNAsmediated crosstalk between OSCC cells and the tumor microenvironment in OSCC metastasis, especially the potential research value of exosomes and lymphangiogenesis, aiming to provide innovative ideas for research related to OSCC metastasis.

\section{THE ROLE OF LnCRNAs IN CANCER METASTASIS}

Studies have shown that lncRNAs can affect tumor metastasis by regulating a variety of biological processes, such as chromatin modification (18), transcription activation $(18,25)$, transcription interference (26), RNA stability regulation (15), and mRNA translation (16), etc. Xu et al. found that lncRNA SATB2-AS1 (antisense transcript of special AT-rich binding protein 2) is lowly expressed in colorectal cancer (CRC) tissues. Further research showed that SATB2-AS1 could directly bind to WDR5 (WD repeat domain 5) and GADD45A (growth arrest and DNA damage-inducible alpha), and then activate SATB2 transcription by mediating the tri-methylation of lysine 4 on histone 3 (H3K4me3) and the DNA demethylation of the promoter region of $\mathrm{SATB} 2$, thus inhibiting the migration, invasion, and metastasis of CRC (18). In contrast, lncRNA CF129 (CF129145.1) was confirmed to inhibit the invasion and metastasis of pancreatic cancer cells by interfering with the transcription of FOXC2 (forkhead box protein C2) (26). Intriguingly, lncRNA BLACAT2 (bladder cancer-associated transcript 2) is also able to bind directly to WDR5, but it promotes lymphangiogenesis and lymphatic metastasis of bladder cancer by enhancing the expression of VEGF-C (vascular endothelial growth factor C) (17). Additionally, $\mathrm{He}$ et al. reported the inhibitory effect of long intergenic noncoding RNA 1093 (LINC01093) on the migration, invasion, and metastasis of liver cancer cells. Mechanistically, by directly binding to IGF2BP1 (insulin-like growth factor 2 mRNAbinding protein 1), LINC01093 could interfere with the interaction between IGF2BP1 and GLI1 (glioma-associated oncogene homolog 1) mRNA, thus leading to the degradation of GLI1 mRNA, which further affects the expression of GLI1 downstream genes involved in the development of liver cancer (15). In addition, in breast cancer, lncRNA RP1 (RP1-5O6.5) was demonstrated to promote the invasion and metastasis of tumor cells through inhibiting the translation of p27kip1 mRNA (16).

Notably, lncRNAs can function as competitive endogenous RNAs (ceRNAs) to sponge miRNAs, blocking their regulation on the expression of their downstream genes, and then inducing various biological effects (27). Moreover, functioning as ceRNAs is also an important mechanism by which lncRNAs regulate 
tumor metastasis. For example, via competitively binding to miR-16-5p, lncRNA AGAP2-AS1 (AGAP2 antisense RNA 1) could weaken the inhibitory effect of miR-16-5p on annexin A11, thereby promoting the migration and invasion of liver cancer cells by enhancing the activation of AKT (protein kinase B) signal pathway (28). Similarly, lncRNA JPX (just proximal to Xist) was indicated to directly target miR-33a-5p, thus upregulating the expression of its downstream gene Twist1, which in turn promotes the metastasis of lung cancer cells through activating the Wnt/ $\beta$-catenin signaling pathway (29).

In general, metastasis is a common feature in the development of many tumors, and the mechanisms of metastasis in different tumors are similar to some extent (30). Therefore, lncRNAs may also regulate the migration, invasion, and lymphangiogenesis of OSCC via (but not limited to) the above mechanisms, thereby inhibiting or promoting the metastasis of OSCC (Table 1).

\section{OVERVIEW OF LnCRNAS INVOLVED IN OSCC METASTASIS}

\section{LncRNAs Regulate Metastasis by Inducing EMT}

Epithelial-mesenchymal transition (EMT) is a process of epithelial phenotype cells differentiating into mesenchymal phenotype cells. In this process, epithelial cells lose polarity and intercellular adhesion and instead acquire the characteristics of migration and invasion (75). Furthermore, the expression levels of some EMT markers will also change significantly during this process, mainly manifested by the decreased expression of E-cadherin (76) and Claudin1 (77), as well as the increased expression of $\beta$-catenin (78), Snail (79) and Vimentin (76), etc. Studies have shown that some lncRNAs regulate the metastasis of OSCC by inducing or inhibiting the EMT process. HOX transcript antisense intergenic RNA (HOTAIR) is a typical example of carcinogenic lncRNAs, which has been found to play a key role in the metastasis of various tumors. For example, in gastric cancer, HOTAIR, functioning as a connecting scaffold, could facilitate the ubiquitination and degradation of Runx3 (runt-related transcription factor 3) by enhancing the interaction between E3 ubiquitin ligase Mex3b (mex-3 RNA binding family member B) and Runx3, thus inhibiting the expression of E-cadherin and Claudin1, and ultimately promoting the migration and invasion of gastric cancer (80). Specifically, HOTAIR has also been shown to be involved in the regulation of OSCC metastasis. Lu et al. revealed that HOTAIR is highly expressed in OSCC tissues, especially in tissues with metastasis. Subsequent studies showed that overexpression of HOTAIR could enhance the metastatic potential of OSCC cells and the expression of EMT markers such as Vimentin, Snail, and Twist. It is worth noting that in clinical samples, HOTAIR is positively correlated with the expression of mesenchymal markers (Snail, Vimentin) and negatively correlated with the expression of epithelial marker (E-cadherin) (41). Additionally, Wu et al.'s research also yielded similar results.
Their data indicated that HOTAIR overexpression is closely related to OSCC metastasis. Moreover, the overexpression of HOTAIR points to lower overall survival and disease-free survival. Mechanistically, HOTAIR is able to recruit histone lysine methyltransferase EZH2 (enhancer of zeste homolog 2) to the promoter region of E-cadherin and then promote H3K27me3 (tri-methylation of lysine 27 on histone 3), thus downregulating the expression of E-cadherin. These data suggest that HOTAIR may regulate the metastasis of OSCC cells by promoting their EMT (42).

In addition to HOTAIR, lncRNA SNHG16 (small nucleolar RNA host gene 16) is also overexpressed in OSCC tissues and can promote the migration and invasion of OSCC cells. Mechanism studies showed that silencing SNHG16 will increase the expression of E-cadherin, and also downregulate the expression of $\mathrm{N}$-cadherin and Snail (58). Interestingly, another lncRNA of the SNHG family, SNHG6 (small nucleolar RNA host gene 6), has also been confirmed to be highly expressed in OSCC tissues, and the expression level of SNHG6 is closely related to lymph node metastasis. Meanwhile, the knockdown of SNHG6 could induce the expression of E-cadherin and inhibit the expression of $\mathrm{N}$-cadherin and Vimentin (59). HNF1A-AS1 (HNF1A antisense RNA 1) is another lncRNA that is highly expressed in OSCC tissues and can promote tumor cell migration and EMT. Mechanistically, HNF1A-AS1 exerts its pro-metastatic effect by activating the Notch signaling pathway (63). In highly metastatic OSCC cells, Li et al. found that the expression of lncRNA AC132217.4 is significantly upregulated. Not only that, but AC132217.4 could also promote the migration and EMT of OSCC cells and accelerate its metastasis. The mechanism is that AC132217.4 interacts with the 3'UTR (3'untranslated region) of IGF2 (insulin-like growth factor 2) mRNA and enhances its stability, thereby enhancing the expression level of IGF2 (61). In another experiment, LINC00460 (long intergenic noncoding RNA 460) was also observed to be highly expressed in OSCC tissues and cells, and its expression level is positively correlated with lymph node metastasis. Further research exhibited the promotion of LINC00460 on the migration, invasion, EMT, and metastasis of OSCC cells. Mechanism research confirmed that there is a direct interaction between LINC00460 and PRDX1 (peroxiredoxin 1) protein, which could facilitate PRDX1 to enter the nucleus. Subsequently, PRDX1 will be enriched in the promoter regions of EMT-related genes, such as ZEB1 (zinc finger $\mathrm{E}$ box binding homeobox 1), ZEB2 (zinc finger $\mathrm{E}$ box binding homeobox 2), and Vimentin, and plays a role in promoting transcription (62). In a nutshell, EMT is a key driving factor for OSCC metastasis, and lncRNAs also play an important role in the EMT of OSCC cells. However, more research is still needed to better understand the mechanisms by which lncRNAs regulate the EMT of OSCC cells.

\section{LncRNAs Regulate Metastasis Through the Wnt/ $\beta$-Catenin Signaling Pathway}

$\mathrm{Wnt} / \beta$-catenin signaling pathway is one of the key pathways regulating tumor development. To date, activation of the Wnt/ $\beta$ catenin signaling pathway has been proved to contribute to the 
TABLE 1 | Regulatory roles of long noncoding RNAs (IncRNAs) in the metastasis-related biological processes of oral squamous cell carcinoma (OSCC).

\begin{tabular}{|c|c|c|c|c|c|}
\hline LncRNA & Expression in OSCC & $\begin{array}{l}\text { Correlation with } \\
\text { tumor metastasis }\end{array}$ & Functions in OSCC & Potential mechanism & Refs \\
\hline $\mathrm{H} 19$ & Overexpressed & Positive & $\uparrow$ Migration, invasion, EMT & CeRNA (let-7a/HMGA2 axis; miR-138/EZH2 axis) & $(31,32)$ \\
\hline LINC00152 & Overexpressed & Positive & $\uparrow$ Migration, invasion, EMT & CeRNA (miR-139-5p; miRNA-193b-3p/PI3K/AKT axis) & $(33,34)$ \\
\hline HOXA11-AS & Overexpressed & - & $\uparrow$ Migration, invasion, EMT & CeRNA (miR-98-5p/YBX2 axis) & (35) \\
\hline GAS5 & Downregulated & - & $\downarrow$ Migration, invasion, EMT & CeRNA (miR-21/PTEN/PI3K/AKT axis) & (36) \\
\hline MALAT1 & Overexpressed & Positive & $\uparrow$ Migration, invasion, EMT & $\begin{array}{l}\text { CeRNA (miR-140-5p/PAK1 axis; miR-124/JAG1 axis); } \\
\text { AKT pathway; } \\
\text { Wnt/ } \beta \text {-catenin pathway; } \\
\text { NF-кB pathway }\end{array}$ & $\begin{array}{c}(21,37) \\
(38) \\
(39) \\
(40)\end{array}$ \\
\hline HOTAIR & Overexpressed & Positive & $\uparrow M i g r a t i o n$, invasion, EMT & $\begin{array}{l}\text { CeRNA (miR-326/MTA2 axis); } \\
\text { Chromatin modification; } \\
\text { SNPs }\end{array}$ & $\begin{array}{c}(41,42) \\
(43) \\
(44)\end{array}$ \\
\hline MEG3 & Downregulated & Negative & $\downarrow$ Migration, invasion & $\begin{array}{l}\text { CeRNA (miR-548d-3p/SOCS5-SOCS6/JAK/STAT axis; miR-21); } \\
\text { Wnt/ß-catenin pathway }\end{array}$ & $\begin{array}{c}(45) \\
(24,46)\end{array}$ \\
\hline AFAP1-AS1 & Overexpressed & - & $\uparrow$ Migration, invasion, EMT & Wnt/ $\beta$-catenin pathway & (23) \\
\hline UCA1 & Overexpressed & Positive & $\uparrow$ Migration, invasion, EMT & $\begin{array}{l}\text { CeRNA (miR-124/TGF- } \beta 1 / J A G 1 / \text { Notch axis; miR-143-3p/MYO6 axis); } \\
\text { Wnt/ } \beta \text {-catenin pathway }\end{array}$ & $\begin{array}{c}(47) \\
(22,48)\end{array}$ \\
\hline TUG1 & Overexpressed & Positive & $\uparrow$ Migration, invasion & $\begin{array}{l}\text { CeRNA (miR-524-5p/DLX1 axis; miR-219/FMNL2 axis); } \\
\text { Wnt/ } \beta \text {-catenin pathway }\end{array}$ & $\begin{array}{c}(49) \\
(50,51)\end{array}$ \\
\hline CASC15 & Overexpressed & - & $\uparrow$ Migration, invasion & CeRNA (miR-33a-5p/ZEB1 axis) & $(52)$ \\
\hline Lnc-p23154 & Overexpressed & Positive & $\uparrow M i g r a t i o n$, invasion, EMT & CeRNA (miR-378a-3p/Glut1 axis) & (53) \\
\hline CCAT1 & Overexpressed & - & $\uparrow$ Migration, invasion & $\begin{array}{l}\text { CeRNA (miR-181a/Wnt/ } \beta \text {-catenin axis); } \\
\text { AKT pathway }\end{array}$ & $\begin{array}{l}(54) \\
(55)\end{array}$ \\
\hline LINC01116 & Overexpressed & Positive & $\uparrow M i g r a t i o n$, invasion, EMT & CeRNA (miR-136/FN1 axis) & $(56)$ \\
\hline SNHG17 & Overexpressed & Positive & $\uparrow$ Migration, invasion & CeRNA (miR-876/SP1 axis) & $(57)$ \\
\hline SNHG16 & Overexpressed & - & $\uparrow M i g r a t i o n$, invasion, EMT & - & $(58)$ \\
\hline SNHG6 & Overexpressed & Positive & $\uparrow E M T$ & - & (59) \\
\hline SNHG3 & Overexpressed & - & $\uparrow$ Migration & By recruiting ELAVL1 to stabilize NFYC mRNA & (60) \\
\hline AC132217.4 & Overexpressed & Positive & $\uparrow$ Migration, EMT & By upregulating IGF2 & $(61)$ \\
\hline LINC00460 & Overexpressed & Positive & $\uparrow M i g r a t i o n$, invasion, EMT & By promoting PRDX1 to enter the nucleus & (62) \\
\hline HNF1A-AS1 & Overexpressed & Positive & $\uparrow M i g r a t i o n$, EMT & Notch pathway & (63) \\
\hline FTH1P3 & Overexpressed & - & $\uparrow$ Migration, invasion & AKT pathway & (64) \\
\hline MIR31HG & Overexpressed & Positive & $\uparrow$ Migration, invasion & By facilitating the formation of HIF-1 complex & $(65)$ \\
\hline HAS2-AS1 & Overexpressed & Positive & $\uparrow I n v a s i o n$, EMT & By enhancing the expression and stability of HAS2 & $(66)$ \\
\hline HOTTIP & Overexpressed & Positive & $\uparrow$ Migration, invasion, EMT & CeRNA (miR-124-3p/HMGA2/Wnt//3-catenin axis) & $(67,68)$ \\
\hline LEF1-AS1 & Overexpressed & - & $\uparrow$ Migration & Hippo pathway & (69) \\
\hline CCAT2 & Overexpressed & Positive & $\uparrow$ Migration, invasion & - & (70) \\
\hline PANDAR & Overexpressed & Positive & $\uparrow$ Migration, invasion & - & $(71)$ \\
\hline NKILA & Downregulated & - & $\downarrow$ Migration, invasion & $N F-\kappa B$ pathway & $(72)$ \\
\hline $\mathrm{RC} 3 \mathrm{H} 2$ & Overexpressed & Positive & $\uparrow$ Migration, invasion & CeRNA (miR-101-3p/EZH2 axis) & (73) \\
\hline TIRY & Overexpressed & - & $\uparrow M i g r a t i o n$, invasion, EMT & CeRNA (miR-14/Wnt/ $\beta$-catenin axis) based on exosomes & $(74)$ \\
\hline
\end{tabular}

metastasis of lung cancer (81), CRC (82), breast cancer (83), and OSCC $(39,45)$. Studies have shown that lncRNAs are important factors in regulating the $\mathrm{Wnt} / \beta$-catenin signaling pathway of OSCC cells. Liu et al. reported that MEG3 is able to inhibit the migration of OSCC cells by weakening the activation of the Wnt/ $\beta$-catenin pathway (45). On the contrary, MALAT1 induces the migration, invasion, and EMT of tongue squamous cell carcinoma (TSCC) cells by activating the $\mathrm{Wnt} / \beta$-catenin pathway (39). As another lncRNA that is highly expressed in OSCC tissue, the expression level of AFAP1-AS1 is negatively correlated with overall survival. Further research confirmed that AFAP1-AS1 is capable of promoting the migration and invasion of OSCC by enhancing the activation of the $\mathrm{Wnt} / \beta$-catenin pathway and the expression of EMT-related genes (23). Yang et al. validated the association between high expression of UCA1 and lymph node metastasis of OSCC. Mechanism research verified that UCA1 could boost the activation of the Wnt/ $\beta$ catenin pathway, thereby promoting the metastasis of OSCC
(47). Similarly, the study by Liang et al. showed that high expression of lncRNA TUG1 (taurine upregulated gene 1) is also significantly associated with lymph node metastasis of OSCC. Furthermore, knocking down TUG1 inhibits the expression of $\beta$-catenin and the invasion ability of tumor cells. However, the use of $\mathrm{LiCl}$ (lithium chloride), a catalyst of the Wnt/ $\beta$-catenin pathway, will weaken the inhibitory effect of TUG1 knockdown on the invasion of OSCC. These data suggest that TUG1 may regulate the migration of OSCC through the Wnt/ $\beta$-catenin pathway (49). Nowadays, specific mechanisms by which lncRNAs regulate the Wnt// $\beta$-catenin signaling pathway remains to be explored.

\section{LncRNAs Regulate Metastasis Through the AKT Signaling Pathway}

AKT signaling pathway is another pathway that has been widely studied and plays an important role in the metastasis of pancreatic cancer (84), gastric cancer (85), lung cancer (86), 
and $\operatorname{OSCC}(38,55)$. In OSCC cells, the AKT signaling pathway is also regulated by various lncRNAs. FTH1P3 (ferritin heavy chain 1 pseudogene 3 ) is a lncRNA that is significantly overexpressed in OSCC tissues. Knocking down FTH1P3 will inhibit the migration and invasion of OSCC cells. Further investigation showed that silencing FTH1P3 contributes to the inhibition of AKT phosphorylation, which may be the mechanism by which FTH1P3 regulates the progression of OSCC (64). Based on the analysis of the expression level of MALAT1 in tissue samples, Yuan et al. reported that the overall survival of TSCC patients with high expression of MALAT1 is significantly lower than that of patients with low expression of MALAT1. Moreover, knocking down MALAT1 will cause the phosphorylation of AKT in TSCC cells to decrease, and also significantly suppress the migration and invasion of TSCC cells, while the application of PI3K (phosphatidylinositol 3-kinase) activator could reverse the inhibitory effect of MALAT1 knockdown on the migration and invasion of TSCC cells, suggesting that MALAT1 may promote the metastasis of TSCC by activating the PI3K/AKT pathway (38). In another experiment, lncRNA CCAT1 (colon cancer-associated transcript 1) was demonstrated to be overexpressed in OSCC cells and be able to promote their migration and invasion. Further mechanistic studies identified that CCAT1 is capable of improving the expression of DDR2 (discoidin domain receptor 2) and directly interacting with it. Subsequently, DDR2 could activate the ERK (extracellular signal-regulated kinase 1)/AKT signaling pathway, thus promoting the invasion and migration of OSCC cells (55). At present, the regulatory role of lncRNAs on the AKT signaling pathway remains to be further explored.

\section{LncRNAs Regulate Metastasis Through the NF-кB Signaling Pathway}

The key role of the NF- $\kappa \mathrm{B}$ signaling pathway in tumor metastasis has been proved by several studies $(87,88)$. Besides, lncRNAs play an important role in the regulation of the NF- $\mathrm{KB}$ signaling pathway. For example, Zheng et al. found that the decreased expression of lncRNA KRT19P3 (Keratin 19 Pseudogene 3) is associated with lymph node metastasis of gastric cancer. Mechanistically, KRT19P3 can directly bind COPS7A and then enhance the stability of COPS7A protein, which subsequently promotes the deubiquitinylation of $\mathrm{I} \kappa \mathrm{B} \alpha$ and then inactivates the NF- $\kappa B$ signaling pathway, thus suppressing tumor metastasis (89). Previous studies have shown that $\operatorname{lncRNAs}$ can also regulate the metastasis of OSCC through the NF- $\kappa \mathrm{B}$ signaling pathway. $\mathrm{Hu}$ et al. showed that lncRNA NKILA (NF- $\mathrm{KB}$ interacting lncRNA) is lowly expressed in OSCC tissues and cells, and the overexpression of NKILA could reduce the invasion and migration of tumor cells, as well as the levels of $I \kappa B \alpha$ and cytoplasm-p65, which indicates that NKILA may regulate the metastasis of OSCC through inhibiting the NF- $\kappa \mathrm{B}$ signaling pathway (72). In contrast, in another study, MALAT1 knockdown was revealed to significantly suppress the levels of NF- $\kappa \mathrm{B}$ and the migration and invasion of OSCC cells (40). However, the mechanism of how lncRNAs regulate OSCC metastasis through the NF- $\mathrm{KB}$ signaling pathway is still unclear.

\section{LncRNAs Regulate Metastasis Through the IL-6/STAT3 Signaling Pathway}

IL-6/STAT3 is an important signaling pathway related to lncRNAs-caused tumor metastasis. Su et al. reported that lncRNA UICC (upregulated in cervical cancer) is highly expressed in cervical cancer tissues, and its high expression is also related to lymph node metastasis. In vitro and in vivo experiments showed that UICC could facilitate the expression of IL- 6 by combining with its promoter and directly interact with the phospho-STAT3 to improve its protein stability, thus promoting tumor growth and metastasis (90). There have been some studies showing that the IL-6/STAT3 signaling pathway plays an important role in the metastasis of OSCC $(91,92)$, but there is no research involving the role of lncRNAs in this process.

\section{LncRNAs Regulate Metastasis by Functioning as ceRNAs MALAT1}

MALAT1 is an evolutionarily highly conserved lncRNA, and its mature transcripts are mainly located in the nucleus, which suggests that MALAT1 may play an important role in the biological processes of cells (93). Recent studies have shown that by regulating chromatin modification (94), gene transcription (95), or acting as ceRNAs (96), MALAT1 is involved in the migration, invasion, and metastasis of tumors. Taking transcription regulation as an example, MALAT1 can recruit $\mathrm{EZH} 2$ to the promoter region of $\mathrm{ABI} 3 \mathrm{BP}$ ( $\mathrm{ABI}$ family member 3 binding protein) and then promote $\mathrm{H} 3 \mathrm{~K} 27 \mathrm{me} 3$, thereby inhibiting the transcription of $\mathrm{ABI} 3 \mathrm{BP}$ and ultimately promoting the metastasis of gallbladder cancer (95). In addition, via directly binding to miR-1914-3p, MALAT1 is capable of enhancing the stability of YAP (Yes-associated protein) mRNA, thus promoting the invasion and metastasis of non-small cell lung cancer (96). Recently, some studies have illustrated that MALAT1 also plays the role of ceRNAs in the regulation of OSCC metastasis. Zhu et al. observed that the expression of MALAT1 in TSCC tissues is significantly increased compared to adjacent non-cancerous tissues. Moreover, high expression of MALAT1 is also closely related to lymph node metastasis. Further experiments showed that after knocking down MALAT1 in TSCC cells, the expression of miR-140-5p is enhanced while the expression of PAK1(p21activated kinase 1) is downregulated. Mechanism studies confirmed the promoting effects of MALAT1 on the expression of PAK1 and the migration and invasion of TSCC by targeting miR-140-5p (21). Apart from that, Zhang et al.'s research also support MALAT1's role of ceRNAs in the regulation of OSCC metastasis. Their data revealed that MALAT1 knockdown could repress the metastasis of TSCC cells in vivo and in vitro by enhancing the expression of JAG1 (jagged 1) through the miR124/JAG1 axis (37).

\section{$\mathrm{H} 19$}

H19 is abnormally expressed in various human cancers, such as pancreatic cancer (97), nasopharyngeal cancer (98), and lung cancer (99), and is usually associated with cancer progression, metastasis, and poor prognosis. Additionally, by acting as 
ceRNAs, H19 has been confirmed to regulate the migration, invasion, and metastasis of various tumors, including nasopharyngeal carcinoma (98), bladder cancer (100), and breast cancer (101). For example, in nasopharyngeal carcinoma, H19 overexpression is closely related to the poor prognosis of patients. Moreover, knocking down H19 could suppress the migration and invasion of tumor cells and delay the progression of transplanted tumor and lung metastasis in nude mice. Mechanistically, H19 upregulates the expression of its downstream proto-oncogene HRAS (HRas proto-oncogene, GTPase) by sponging miRNA let-7, thus promoting the carcinogenic activity of HRAS in nasopharyngeal carcinoma (98). Similarly, the interaction between $\mathrm{H} 19$ and various miRNAs is also an important mechanism for its regulation of OSCC metastasis. Kou et al. found that the expression of H19 in metastatic TSCC tissues is higher than that of non-metastatic TSCC tissues, and H19 knockdown could attenuate the migration and invasion of TSCC cells. The speculated mechanism of which is that $\mathrm{H} 19$ potentiates the expression of HMGA2 (high mobility group AT-hook 2), a key regulator of tumor metastasis, by targeting let-7. Conversely, inhibition of let7a will dampen the inhibitory effect of H19 knockdown on the migration and invasion of TSCC cells (31). Another study revealed that the expression of $\mathrm{H} 19$ is positively correlated with the TNM stage and negatively related to overall survival. Further data implied that H19 can enhance the expression of its target gene EZH2 by sponging miR-138, thereby promoting the migration, invasion, and EMT of OSCC cells (32).

\section{MEG3}

Unlike MALAT1 and H19, MEG3 usually plays the role of a tumor suppressor. Numerous studies have confirmed that MEG3 is lowly expressed in various tumors, such as CRC (102), bladder cancer (103), and renal cell cancer (104), and inhibits their migration, invasion, and metastasis. Zhu et al. reported that MEG3 is downregulated in CRC tissues. In addition, overexpression of MEG3 could suppress the migration, invasion, and metastasis of tumor cells by inhibiting the expression of clusterin (102). A study on bladder cancer validated that by competing with PHLPP2 (PH domain and leucine-rich repeat protein phosphatase 2) mRNA to bind miR27a, thereby promoting the expression of PHLPP2, MEG3 can restrain the invasion and metastasis of bladder cancer cells (103). Similarly, MEG3 can also suppress the migration and invasion of OSCC by exerting the function of ceRNAs. Tan et al. proved the low expression of MEG3 in OSCC tissues. Moreover, overexpression of MEG3 contributes to the downregulation of miR-548d-3p as well as the migration and invasion of OSCC cells. Mechanistically, the inhibitory effect of MEG3 on miR548d-3p enhances the expression of miR-548d-3p downstream targets SOCS5 (suppressor of cytokine signaling 5) and SOCS6 (suppressor of cytokine signaling 6). Next, SOCS5/SOCS6 could repress the migration and invasion of OSCC by weakening the activation of the JAK/STAT (janus kinase/signal transducer and activator of transcription) pathway (24). Another study reported that MEG3 in OSCC tissue is negatively correlated with miR-21. Subsequent dual luciferase assay identified the direct interaction between MEG3 and miR-21. Furthermore, inhibiting the expression of miR-21 will reduce the migration ability of OSCC cells, and knocking down MEG3 could partially reverse the inhibitory effect of miR-21 downregulation on the migration of OSCC cells (46), which implies that MEG3 may dampen the migration of OSCC by targeting miR-21, but its downstream molecular mechanisms need further investigation. At the same time, more in vivo experiments are desired to clarify the role of MEG3 in OSCC metastasis.

\section{LINC00152}

LINC00152 (long intergenic noncoding RNA 152), transcribed from the region between protein-coding genes PLGLB2 (plasminogen like B2) and PLGLB1 (plasminogen like B1), is also known as CYTOR (cytoskeleton regulator RNA). Through interfering with cell signaling pathways such as PI3K/AKT (105), FSCN1 (fascin actin-bundling protein 1) (106), and Wnt/ $\beta$ catenin $(107,108)$, LINC00152 was demonstrated to promote the invasion and metastasis of diverse tumors. At present, research on the function of LINC00152 in OSCC metastasis is also mainly focused on its role of ceRNAs. By analyzing two sets of TSCC gene expression profile data (GSE30784 and GSE9844), $\mathrm{Yu}$ et al. found that LINC00152 is significantly upregulated in TSCC tissues, and this result was also confirmed in fresh specimens and paraffin-embedded specimens. Further analysis showed that high expression of LINC00152 is closely related to lymph node metastasis of TSCC (109). Similarly, Li et al. validated that LINC00152 is highly expressed in OSCC tissues and cells. Moreover, knocking down LINC00152 could restrain the migration, invasion, and EMT of OSCC cells in vitro. Mechanistically, LINC00152 might play a role in promoting cancer by competitively binding miR-139-5p (33). Beyond that, miRNA-193b-3p is also a target of LINC00152. Further data implied that the inhibitory effect of LINC00152 on miRNA$193 \mathrm{~b}-3 \mathrm{p}$ is responsible for the activation of the PI3K/AKT signaling pathway, which contributes to the migration and invasion of TSCC in vitro (34). However, more in vivo experiments are still needed to further illustrate the impact of LINC00152 on OSCC metastasis.

\section{UCA1}

UCA1 was first discovered in bladder cancer (110) and has been proved to promote the migration and invasion of bladder cancer cells (111). Not only that, but UCA1 can also regulate the metastasis of gastric cancer (112), osteosarcoma (113), and breast cancer (114), etc. Taking gastric cancer as an example, Gong et al. confirmed that UCA1 expression is abnormally increased in gastric cancer tissues, and high expression of UCA1 is significantly associated with lymph node metastasis. Additionally, overexpression of UCA1 was demonstrated to promote the migration, invasion, and metastasis of gastric cancer cells. The mechanism is that the direct interaction between UCA1 and miR-203 enhances the expression of miR203 target ZEB2 (112). Recently, abnormal expression of UCA1 has also been found to be associated with OSCC metastasis. Fang et al. observed that UCA1 is highly expressed in TSCC tissues, and its expression level is positively correlated with lymph node 
metastasis (115). Similarly, Zhang et al. also identified the correlation between UCA1 overexpression and the poor prognosis (lymph node metastasis and shorter survival time) in patients with TSCC. Interestingly, in TSCC cells, UCA1 can sponge miR-124 and negatively regulate each other. Further research showed that by targeting miR-124 to increase the expression of TGF- $\beta 1$ (transforming growth factor $\beta 1$ ), UCA1 can activate the JAG1/Notch pathway, which in turn promotes invasion and EMT of TSCC cells (22). Moreover, in another experiment, UCA1 was found to competitively bind miR-143-3p with MYO6 (myosin VI), thus promoting the migration, invasion, and EMT of TSCC cells (48).

\section{TUG1}

TUG1 is another lncRNA that has been extensively explored. One study proved that TUG1 plays a role in promoting the metastasis of CRC via upregulating the expression of Twist1 (116). Apart from that, by competitively binding miR-143-5p, TUG1 could enhance the expression of HIF-1 $\alpha$ (hypoxia inducible factor 1 subunit alpha), thereby facilitating the metastasis of osteosarcoma cells (117). Similarly, the chain reaction caused by the direct binding of TUG1 to miR-455-3p also contributes to liver cancer metastasis (118). In particular, TUG1 was unveiled to promote the metastasis of OSCC by weakening the inhibitory effect of miRNAs on their downstream targets. Liu et al. confirmed that TUG1 is highly expressed in OSCC cells, and can enhance the migration potential of OSCC cells. The mechanism may be that TUG1 competitively binds miR-524-5p with DLX1(distal-less homeobox 1) and thus improves the expression of DLX1 (50). Apart from that, Yan et al. discovered that TUG1 knockdown could suppress the migration and invasion of OSCC cells, while overexpression of TUG1 will boost the metastasis of OSCC in vivo. Further experiments verified that there is mutual inhibition between TUG1 and miR-219, and repression of miR-219 could reverse the inhibitory effect of TUG1 knockdown on OSCC cells. Mechanistically, by acting as a ceRNA to sponge miR-219, TUG1 can enhance the expression of miR-219 target FMNL2 (formin-like protein 2) and thereby exerting its pro-metastasis effect (51).

\section{Other LncRNAs Functioning as ceRNAs in OSCC Metastasis}

LncRNA HOXA11-AS (homeobox A11 antisense RNA) was found to be highly expressed in OSCC tissues and cells. Moreover, HOXA11-AS was demonstrated to trigger the expression of YBX2 (Y box binding protein 2) by targeting miR-98-5p, thus promoting the migration, invasion, and EMT of OSCC cells (35). In contrast, lncRNA GAS5 (growth-arrestspecific transcript 5) is lowly expressed in OSCC. Furthermore, upregulating the expression of GAS5 could inhibit the migration, invasion, and EMT of OSCC cells, and the mechanism is also to play the role of ceRNA. Specifically, GAS5 enhances the expression of PTEN (phosphatase and tensin homolog) by combining with miR-21, and then dampened the activation of the PI3K/AKT signaling pathway. Beyond that, the inhibition of
miR-21 by GAS5 also increased the expression of E-cadherin, while decreased the expression of $\mathrm{N}$-cadherin, Vimentin, and Snail, suggesting that the EMT of OSCC cells was repressed as well (36). The results of another study showed that HOTAIR can improve the expression of MTA2 (metastasis-associated gene 2) via the miR-326/MTA2 axis, thereby facilitating the migration, invasion, and EMT of OSCC cells (43). Similarly, lncRNA CASC15 (cancer susceptibility candidate 15) has also played a role in promoting metastasis in the development of OSCC. The mechanism may be that the combination of CASC15 and miR33a-5p elevates the expression level of the downstream target ZEB1 of miR-33a-5p (52). Wang et al. found that lncRNA lncp23154 is highly expressed in OSCC tissues and cells. Mechanism studies confirmed that by directly binding to miR$378 \mathrm{a}-3 \mathrm{p}$, lnc-p23154 is capable of upregulating the expression of Glut1 (glucose transporter 1), thereby reinforcing the glycolysis of OSCC cells, and ultimately inducing the migration, invasion, EMT, and metastasis of OSCC cells (53). Additionally, lncRNAs CCAT1 (54), LINC01116 (long intergenic noncoding RNA 1162) (56), SNHG17 (small nucleolar RNA host gene 17) (57), and $\mathrm{RC} 3 \mathrm{H} 2$ (73) have been proved to facilitate the metastasisrelated biological behaviors of OSCC cells via regulating miR181a/Wnt/ß-catenin, miR-136/FN1 (fibronectin1), miR-876/SP1 (specificity protein 1), and miR-101-3p/EZH2 signaling pathways respectively (Figure 1). In a word, lncRNAs play a key role in the metastasis of OSCC by acting as ceRNAs.

\section{LncRNAs Involved in the Distant Metastasis of OSCC}

The probability of distant metastasis occurring in patients with OSCC is relatively low, only about $10 \%(119,120)$. However, once a distant metastasis occurs, the patient's hope of being cured is very slim. Studies have unveiled that lncRNAs participate in regulating the distant metastasis of various tumors including OSCC. Wang et al. found that lncRNA APC1 (adenomatous polyposis coli 1) is lowly expressed in CRC tissues, and its expression level is negatively correlated with distant metastasis. In addition, in a nude mouse model, the overexpression of APC1 could attenuate lung metastasis of CRC cells. The mechanism may be that APC1 directly binds to Rab5b mRNA and reduces its stability, thereby inhibiting the production of exosomes derived from tumor cells (121). In contrast, lncRNA BX11 (BX111887) is overexpressed in pancreatic cancer, and its expression level is positively related to distant metastasis. Further research confirmed that by recruiting the transcription factor $\mathrm{YB} 1$ (Y-box binding protein 1 ) to the promoter region of $Z E B 1, B X 11$ is able to activate the transcription of ZEB1, thus promoting the distant metastasis of tumor cells (122).

Currently, there are few studies on the role of lncRNAs in the distant metastasis of OSCC. LncRNA HOTTIP (HOXA transcript at the distal tip) was reported to be overexpressed in TSCC tissues, and its high expression is associated with distant metastasis (120). Mu et al. observed that knocking down HOTTIP could restrain the migration, invasion, and EMT of TSCC cells (67). Moreover, the results of another study identified 


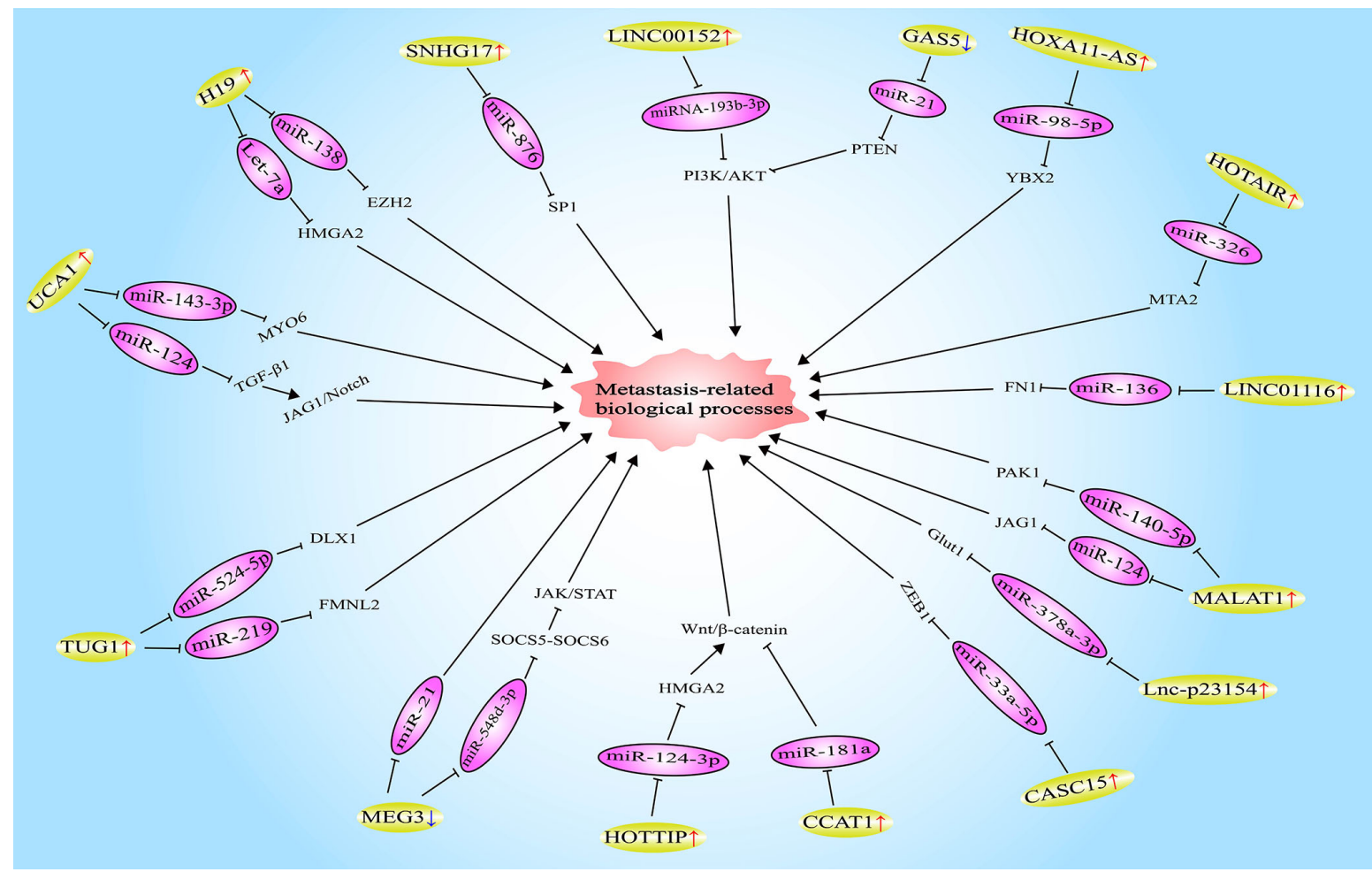

FIGURE 1 | Long noncoding RNAs (LncRNAs) involved in oral squamous cell carcinoma (OSCC) metastasis by functioning as competitive endogenous RNAs (ceRNAs).

that HOTTIP is upregulated in TSCC tissues with lymph node metastasis compared to those without lymph node metastasis. Mechanism research showed that by targeting miR-124-3p, HOTTIP is capable of enhancing the expression of HMGA2 (high mobility group AT-hook 2), which will further activate the $\mathrm{Wnt} / \beta$-catenin pathway and promote the migration and invasion of TSCC cells (68). However, potential mechanisms of HOTTIP to regulate the distant metastasis of OSCC remain to be studied. In addition to HOTTIP, the high expression of lncRNA CCAT2 (colon cancer-associated transcript 2) (70) and lncRNA PANDAR (promoter of CDKN1A antisense DNA damage activated RNA) (71) are all associated with the distant metastasis of OSCC. Furthermore, both knockdowns of CCAT2 and PANDAR could inhibit the migration and invasion of OSCC cells. Whereas, the mechanisms by which CCAT2 and PANDAR regulate the distant metastasis of OSCC are still unclear.

\section{Other Potential Mechanisms of LncRNAs Regulating the Metastasis of OSCC}

To evaluate the influence of HOTAIR gene polymorphism and environmental factors on the susceptibility of OSCC, Su et al. detected four single-nucleotide polymorphisms (SNPs) of HOTAIR, namely rs920778, rs1899663, rs4759314, and rs12427129, in 1200 control participants and 907 OSCC patients. They found that rs920778 is associated with a high rate of lymph node metastasis. In addition, a motif search on the HOTAIR enhancer region showed that rs920778 is located in the putative binding motif of PRDM14 (PRDI-BF1 and RIZ domain containing 14). In particular, PRDM14 is a transcription factor that plays an anti-cancer role in HPV-positive OSCC cells (123). Moreover, data from the GTEx (gnotype-tissue expression) database shows that HOTAIR is differentially expressed in muscle skeletal tissues of individuals carrying the polymorphic allele of rs920778. These results suggest that the alteration in HOTAIR expression caused by SNPs might affect the development of OSCC, and this change may come from abnormal regulation of HOTAIR expression caused by SNPs, the mechanisms of which needs to be further explored (44).

Studies have validated that in a hypoxic environment, some lncRNAs could accelerate the metastasis of OSCC. Shih et al. reported that the expression of lncRNA MIR31HG (miR-31 host gene) in OSCC tissues is increased and can be induced by hypoxia. In addition, MIR31HG overexpression is also closely related to lymph node metastasis. Furthermore, MIR31HG in the hypoxic environment has a more obvious promotion effect on the migration, invasion, and metastasis of OSCC cells, compared with that in the normoxic environment. Mechanism studies confirmed that MIR31HG is capable of promoting the transcription of HIF- $1 \alpha$ and bind to it, thereby assisting its binding to HIF-1 $\beta$ (hypoxia inducible factor 1 subunit beta) and histone acetylase p300, thus forming the HIF-1 complex. 
Apart from that, MIR31HG is also able to recruit the HIF-1 complex to the promoter region of the metastasis-promoting genes, such as VEGF (vascular endothelial growth factor), LICAM(L1 cell adhesion molecule), and LOXL2 (lysyloxidase homologe2), to activate their transcription (65). Similarly, lncRNA HAS2-AS1 (hyaluronan synthase 2 antisense 1) is highly expressed in OSCC tissues and cells under hypoxic conditions, and HAS2-AS1 overexpression is also closely associated with lymph node metastasis. Subsequent studies exhibited that hypoxiainduced overexpression of HAS2-AS1 depends on HIF-1 $\alpha$, which can directly bind to the promoter region of HAS2-AS1 and activate its transcription. Additionally, HAS2-AS1 was proved to promote the hypoxia-induced invasion and EMT of OSCC cells. The potential mechanism is that HAS2-AS1 increases the level of hyaluronic acid in OSCC cells by enhancing the expression and stability of HAS2 (hyaluronan synthase 2) (66).

LncRNA SNHG3 (small nucleolar RNA host gene 3) was found to be overexpressed in OSCC cells. Silencing SNHG3 will inhibit the migration ability of OSCC cells. Further research showed that by recruiting ELAVL1 (ELAV like RNA-binding protein 1) to bind with NFYC (nuclear transcription factor Y subunit gamma) mRNA and then enhance its stability, SNHG3 could increase the expression of NFYC and thereby induce the migration of OSCC cells (60). As another lncRNA highly expressed in OSCC tissues and cells, LEF1-AS1 (lymphoid enhancer-binding factor 1 antisense RNA 1) has been validated to inhibit the activation of the Hippo pathway by direct interaction with LATS1 (large tumor suppressor 1), thus promoting the migration of OSCC cells (69). In general, mechanisms by which lncRNAs regulate OSCC metastasis are complex. One lncRNA could regulate OSCC metastasis through multiple mechanisms, while diverse lncRNAs acting on the same signaling pathway may produce opposite effects. To fully understand the biological function of lncRNAs, especially its role in OSCC metastasis, there are still many studies to be carried out.

\section{THE REGULATION OF CAFs-RELATED LNCRNAS ON THE METASTASIS OF OSCC}

In recent years, the tumor microenvironment composed of cells (such as fibroblasts, endothelial cells, and immune cells) and extracellular components (such as growth factors, hormones, and extracellular matrix) has become a hot spot in tumor research (124). Studies have confirmed that the heterogeneity of the tumor microenvironment contributes to the occurrence and metastasis of tumors $(125,126)$. Cancer-associated fibroblasts (CAFs) are an important component of the tumor microenvironment. The interaction of CAFs with tumor cells not only plays an important part in mediating the formation of CAFs (127) but also affects the process of tumor metastasis (128). It interests to note that lncRNAs participates in the regulation of CAFs on the metastasis of tumors (Figure 2). Ren et al. reported that high expression of HOTAIR is associated with breast cancer metastasis. In addition, the conditioned medium of CAFsderived from breast cancer could significantly enhance the expression of HOTAIR and promote the metastasis of breast cancer cells. Subsequently, mechanism research validated the promoting effect of TGF- $\beta 1$ secreted by CAFs on the transcription of HOTAIR in breast cancer cells, which will enhance the expression of CDK5 (cyclin dependent kinase 5), thereby facilitating the metastasis of breast cancer cells (129). In another experiment, lncRNA LINC00092 (long intergenic noncoding RNA 92) was found to be highly expressed in ovarian cancer tissues, and its overexpression is associated with tumor metastasis. Further investigation showed that CXCL14 (C-X-C motif chemokine ligand 14) derived from CAFs could increase the expression of LINC00092 in ovarian cancer cells. Moreover, LINC00092 was demonstrated to promote the glycolysis of tumor cells and induce the metastasis of ovarian cancer cells by enhancing the expression of glycolytic enzyme PFKFB2 (6-phosphofructo-2-kinase/fructose-2,6bisphosphatase 2) and combining with it (130). In addition to regulating the expression of lncRNAs in tumor cells, CAFs may also regulate tumor metastasis by delivering lncRNAs to tumor cells. Ren et al. confirmed that CAFs derived from CRC are capable of transferring $\mathrm{H} 19$ to CRC cells through exosomes, thereby increasing the level of $\mathrm{H} 19$ in tumor cells and promoting the proliferation of tumor cells. The mechanism is that the direct binding of $\mathrm{H} 19$ to $\mathrm{miR}-141$ activates the $\mathrm{Wnt} / \beta$-catenin signaling pathway in tumor cells (131). As mentioned earlier, the activation of the $\mathrm{Wnt} / \beta$-catenin signaling pathway has been shown to promote the metastasis of CRC (82). Besides, H19 overexpression is also an important factor inducing CRC metastasis (132). However, further research is needed to confirm whether CAFs-derived $\mathrm{H} 19$ can promote the metastasis of CRC.

Currently, only one study has explored the role of lncRNAs in the regulation of CAFs on the metastasis of OSCC. Jin et al. observed that lncRNA TIRY is highly expressed in CAFs derived from OSCC tissues. Furthermore, after co-cultivation of OSCC cells with conditioned medium of CAFs treated with TIRY overexpression plasmids, the capabilities of migration and invasion of OSCC cells were significantly improved. Moreover, knocking down TIRY caused increased expression of miR-14 in CAFs and their exosomes, whose mechanism is the direct binding of TIRY to miR-14. Further research showed that the overexpression of TIRY inhibits the transmission of miR-14 from CAFs to tumor cells through the transport of exosomes, thus enhancing the activation of the $\mathrm{Wnt} / \beta$-catenin signaling pathway in tumor cells and promoting EMT. This may be the mechanism by which TIRY in CAFs regulates the migration and invasion of OSCC cells. Interestingly, the expression of miR-14 in OSCC cells was substantially increased after treatment with conditioned medium of TIRY-silenced CAFs (74). However, it is still unknown whether TIRY can be directly transported into OSCC cells through CAFs-derived exosomes and then affect the metastasis of OSCC. At present, research on the role of CAFsrelated lncRNAs in OSCC metastasis has just begun, and there is still much work to be done. 
A

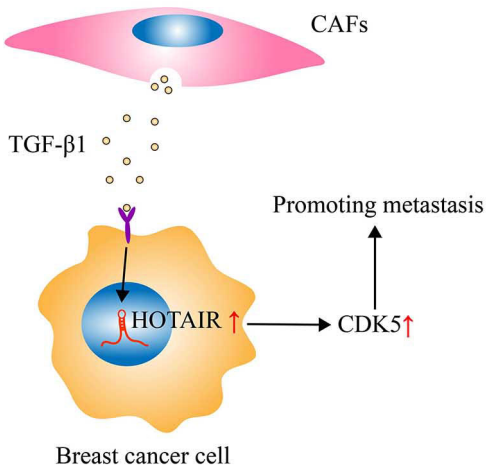

B

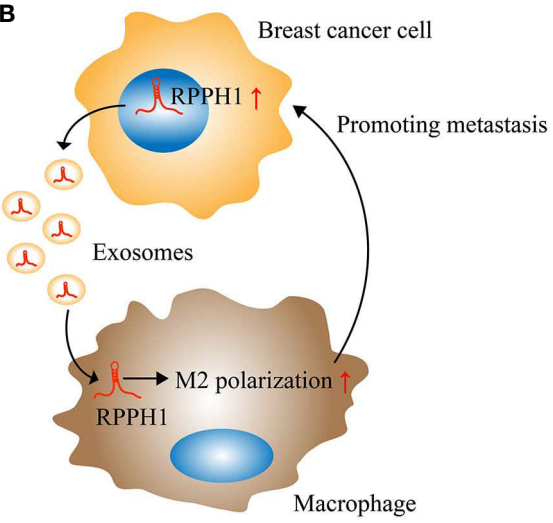

C Exosomes

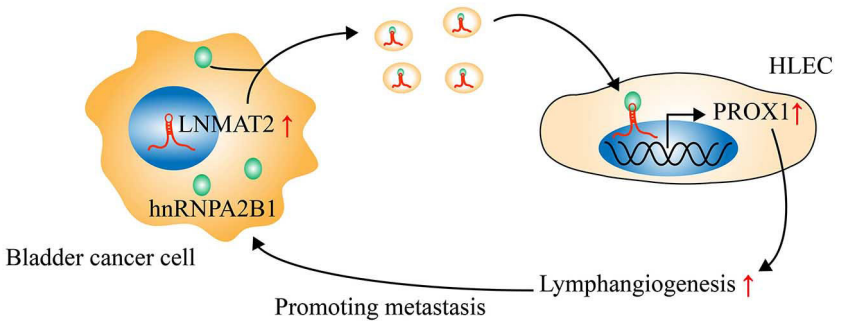

FIGURE 2 | Long noncoding RNAs (LncRNAs) regulate tumor metastasis by mediating the crosstalk between tumor cells and tumor microenvironment. (A) Cancerassociated fibroblasts (CAFs) derived from breast cancer could secrete TGF- $\beta 1$ to activate the transcription of HOX transcript antisense intergenic RNA (HOTAIR) in breast cancer cells, thereby enhancing the expression of CDK5 and then inducing metastasis. (B) Through exosomes, RPPH1 derived from breast cancer cells could be transported into macrophages and then induce M2 polarization of macrophages, which will further accelerate the metastasis of breast cancer. (C) LNMAT2 could be encapsulated into the exosomes secreted by bladder cancer cells through direct interaction with hnRNPA2B1. Subsequently, the exosomal LNMAT2 internalized by human lymphatic endothelial cell (HLEC) could promote the expression of PROX1 in HLEC, thus leading to lymphangiogenesis and lymph node metastasis.

\section{CONCLUSION}

Research in the past decade has partially revealed the key role played by $\ln$ RRNAs in the development of OSCC. In particular, abnormally expressed lncRNAs are also important factors affecting OSCC metastasis. LncRNAs can regulate the metastasis of OSCC through different mechanisms at epigenetic (18), transcription (26), and post-transcriptional (16) levels, and play the role of oncogene or tumor suppressor. Nonetheless, the current focus of scholars is mainly on the effect of abnormal expression of autologous lncRNAs in OSCC cells on tumor metastasis. The role of lncRNAs-mediated crosstalk between OSCC cells and the tumor microenvironment in tumor metastasis is still seldom studied. However, the stromal cells (such as CAFs (129), endothelial cells (133) and immune cells (134)), exosomes (135), and lymphangiogenesis (133) involved in this crosstalk have been shown to be involved in the metastasis of other tumors (Figure 2).

As mentioned previously, based on the crosstalk between CAFs and tumor cells, CAFs-related lncRNAs play an important role in tumor metastasis. It is worth noting that exosomes can act as messengers between tumor cells and CAFs. Moreover, exosomes are also the key link between tumor cells and other stromal cells, which contribute to the metastasis of various tumors as well $(133,134)$. The regulation of exosomes on tumor metastasis depends on their special structure (membranous microcapsules with diameters ranging from 50 to $150 \mathrm{~nm}$ ) and contents (RNA, DNA, proteins, etc.) (136). Previous studies have identified that exosomes could transfer lncRNAs to target cells and induce a series of chain reactions, thereby regulating tumor metastasis $(134,135)$. For example, Liang et al. found that the expression of lncRNA RPPH1 (ribonuclease P RNA component $\mathrm{H} 1$ ) in breast cancer tissues is significantly upregulated. Moreover, $\mathrm{RPPH} 1$ overexpression is associated with metastasis and poor prognosis. In addition, the in vivo study demonstrated that RPPH1 is able to promote the metastasis of breast cancer. Mechanistically, through exosomes transport into macrophages, RPPH1 derived from breast cancer cells could induce M2 polarization of macrophages. In turn, M2polarized macrophages will further accelerate breast cancer metastasis (134). However, there is currently no study on the effect of exosomal lncRNAs on OSCC metastasis. Therefore, further exploration of the role of exosomal lncRNAs in OSCC metastasis will provide more valuable information on the metastasis of OSCC. 
The high rate of lymph node metastasis is the main cause of death in patients with OSCC (5). Not only OSCC, but many other tumors can also have lymph node metastasis $(112,133$, 134). As an important channel for lymph node metastasis of tumor cells, lymphatic vessels and the mechanisms of their generation naturally attract the attention of scholars. Studies have confirmed that lncRNAs are involved in inducing lymphangiogenesis of tumors $(133,137)$. Taking lncRNA LNMAT2 (lymph node metastasis-associated transcript 2) as an example (133), Chen et al. found that bladder cancer-derived exosomal LNMAT2 can enhance the ability of tube formation and migration of human lymphatic endothelial cells (HLEC) in vitro, and facilitate tumor lymphangiogenesis and lymph node metastasis in vivo. Further mechanistic studies uncovered that LNMAT2 could be encapsulated into exosomes secreted by bladder cancer cells through direct interaction with hnRNPA2B1 (heterogeneous nuclear ribonucleoprotein A2B1). Subsequently, the exosomal LNMAT2 will be internalized by HLEC, and then promote the expression of PROX1 (prospero homeobox 1) at the epigenetic level, thus leading to lymphangiogenesis and lymph node metastasis of the tumor. Therefore, LNMAT2 has the potential to serve as a therapeutic target for lymph node metastasis of bladder cancer. However, to date, there has been no research on the regulation of lncRNAs on OSCC lymphangiogenesis. Subsequent research should pay more attention to this direction.

In addition to the crosstalk between tumor cells and stromal cells, the crosstalk between tumor cells and the extracellular matrix (ECM) also plays a critical role in the metastasis of tumors. Huang et al. reported that integrin alpha 2 (ITGA2), upregulated in omental metastases of patients with ovarian cancer, can trigger tumor cell adhesion to collagen, and then facilitates the migration and peritoneal metastasis of tumor cells. The speculated mechanism of which is that ITGA2 activates the phosphorylation of focal adhesion kinase and the mitogen-

\section{REFERENCES}

1. Ketabat F, Pundir M, Mohabatpour F, Lobanova L, Koutsopoulos S, Hadjiiski L, et al. Controlled Drug Delivery Systems for Oral Cancer Treatment-Current Status and Future Perspectives. Pharmaceutics (2019) 11(7):302. doi: 10.3390/pharmaceutics11070302

2. Torre LA, Bray F, Siegel RL, Ferlay J, Lortet-Tieulent J, Jemal A. Global cancer statistics, 2012. CA Cancer J Clin (2015) 65(2):87-108. doi: 10.3322/ caac. 21262

3. Ganly I, Patel S, Shah J. Early stage squamous cell cancer of the oral tongueclinicopathologic features affecting outcome. Cancer (2012) 118(1):101-11. doi: $10.1002 /$ cncr.26229

4. Alves AM, Diel LF, Lamers ML. Macrophages and prognosis of oral squamous cell carcinoma: A systematic review. J Oral Pathol Med (2018) 47(5):460-7. doi: 10.1111/jop.12643

5. Zanoni DK, Montero PH, Migliacci JC, Shah JP, Wong RJ, Ganly I, et al. Survival outcomes after treatment of cancer of the oral cavity (1985-2015). Oral Oncol (2019) 90:115-21. doi: 10.1016/j.oraloncology.2019.02.001

6. Romer CAE, Broglie Daeppen MA, Mueller M, Huber GF, Guesewell S, Stoeckli SJ. Long-term speech and swallowing function after primary resection and sentinel node biopsy for early oral squamous cell carcinoma. Oral Oncol (2019) 89:127-32. doi: 10.1016/j.oraloncology.2018.12.027 activated protein kinase pathway (138). In another study, lncRNA CASC9 (cancer susceptibility 9) was revealed to be associated with the prognosis and metastasis of esophageal squamous cell carcinoma (ESCC). Besides, CASC9 knockdown could significantly inhibit the migration, invasion, and metastasis of ESCC cells. Mechanistically, CASC9 can enhance the enrichment of the transcriptional coactivator CREB-binding protein and H3K27 acetylation in the promoter of LAMC2, an upstream inducer of the integrin pathway, thus promoting its expression. Nevertheless, the impact of the crosstalk between tumor cells and ECM regulated by lncRNAs on OSCC metastasis has not been studied.

In summary, lncRNAs are expected to serve as indicators for screening, treatment, and prognostic monitoring of OSCC metastasis. However, the current understanding of the role of lncRNAs in OSCC metastasis is still in its infancy. Therefore, further research on the role of lncRNAs in the crosstalk between OSCC cells and tumor microenvironment will help enrich the blueprint of the mechanisms of OSCC metastasis.

\section{AUTHOR CONTRIBUTIONS}

YX and EJ discussed and designed the study. YX performed the research and analyzed the data. ZS and ZJS revised the article. All authors contributed to the article and approved the submitted version.

\section{FUNDING}

This study was supported by grants from the National Natural Science Foundation of China 81772897, 81672666.

7. Guo CB, Feng Z, Zhang JG, Peng X, Cai ZG, Mao C, et al. Supraomohyoid neck dissection and modified radical neck dissection for clinically nodenegative oral squamous cell carcinoma: a prospective study of prognosis, complications and quality of life. J Craniomaxillofac Surg (2014) 42 (8):1885-90. doi: 10.1016/j.jcms.2014.07.007

8. Moratin J, Metzger K, Kansy K, Ristow O, Engel M, Hoffmann J, et al. The prognostic significance of the lymph node ratio in oral cancer differs for anatomical subsites. Int J Oral Maxillofac Surg (2020) 49(5):558-63. doi: 10.1016/j.ijom.2019.10.015

9. Todorović V, Desai BV, Eigenheer RA, Yin T, Amargo EV, Mrksich M, et al. Detection of differentially expressed basal cell proteins by mass spectrometry. Mol Cell Proteomics (2010) 9(2):351-61. doi: 10.1074/ mcp.M900358-MCP200

10. Sasahira T, Kirita T, Kurihara M, Yamamoto K, Bhawal UK, Bosserhoff AK, et al. MIA-dependent angiogenesis and lymphangiogenesis are closely associated with progression, nodal metastasis and poor prognosis in tongue squamous cell carcinoma. Eur J Cancer (2010) 46(12):2285-94. doi: 10.1016/j.ejca.2010.04.027

11. Slavoff SA, Mitchell AJ, Schwaid AG, Cabili MN, Ma J, Levin JZ, et al. Peptidomic discovery of short open reading frame-encoded peptides in human cells. Nat Chem Biol (2013) 9(1):59-64. doi: 10.1038/ nchembio. 1120 
12. Iyer MK, Niknafs YS, Malik R, Singhal U, Sahu A, Hosono Y, et al. The landscape of long noncoding RNAs in the human transcriptome. Nat Genet (2015) 47(3):199-208. doi: 10.1038/ng.3192

13. Yan X, Hu Z, Feng Y, Hu X, Yuan J, Zhao SD, et al. Comprehensive Genomic Characterization of Long Non-coding RNAs across Human Cancers. Cancer Cell (2015) 28(4):529-40. doi: 10.1016/j.ccell.2015.09.006

14. Begolli R, Sideris N, Giakountis A. LncRNAs as Chromatin Regulators in Cancer: From Molecular Function to Clinical Potential. Cancers (Basel) (2019) 11(10)):1524. doi: 10.3390/cancers11101524

15. He J, Zuo Q, Hu B, Jin H, Wang C, Cheng Z, et al. A novel, liver-specific long noncoding RNA LINC01093 suppresses HCC progression by interaction with IGF2BP1 to facilitate decay of GLI1 mRNA. Cancer Lett (2019) 450:98109. doi: 10.1016/j.canlet.2019.02.033

16. Jia X, Shi L, Wang X, Luo L, Ling L, Yin J, et al. KLF5 regulated lncRNA $\mathrm{RP} 1$ promotes the growth and metastasis of breast cancer via repressing p27kip1 translation. Cell Death Dis (2019) 10(5):373. doi: 10.1038/s41419019-1566-5

17. He W, Zhong G, Jiang N, Wang B, Fan X, Chen C, et al. Long noncoding RNA BLACAT2 promotes bladder cancer-associated lymphangiogenesis and lymphatic metastasis. J Clin Invest (2018) 128(2):861-75. doi: $10.1172 /$ jci96218

18. Xu M, Xu X, Pan B, Chen X, Lin K, Zeng K, et al. LncRNA SATB2-AS1 inhibits tumor metastasis and affects the tumor immune cell microenvironment in colorectal cancer by regulating SATB2. Mol Cancer (2019) 18(1):135. doi: 10.1186/s12943-019-1063-6

19. Zhou S, Zhu Y, He Z, Zhang D, Guo F, Jian X, et al. Long Non-Coding RNA Expression Profile Associated with Malignant Progression of Oral Submucous Fibrosis. J Oncol (2019) 2019:6835176. doi: 10.1155/2019/ 6835176

20. Cao W, Liu JN, Liu Z, Wang X, Han ZG, Ji T, et al. A three-lncRNA signature derived from the Atlas of ncRNA in cancer (TANRIC) database predicts the survival of patients with head and neck squamous cell carcinoma. Oral Oncol (2017) 65:94-101. doi: 10.1016/j.oraloncology.2016.12.017

21. Zhu MH, Zhang CY, Chen DH, Chen SC, Zheng HL. IncRNA MALAT1 potentiates the progression of tongue squamous cell carcinoma through regulating miR-140-5p-PAK1 pathway. Onco Targets Ther (2019) 12:136577. doi: 10.2147/ott.S192069

22. Zhang TH, Liang LZ, Liu XL, Wu JN, Su K, Chen JY, et al. LncRNA UCA1/ miR-124 axis modulates TGF1-induced epithelial-mesenchymal transition and invasion of tongue cancer cells through JAG1/Notch signaling. J Cell Biochem (2019) 120(6):10495-504. doi: 10.1002/jcb.28334

23. Wang ZY, Hu M, Dai MH, Xiong J, Zhang S, Wu HJ, et al. Upregulation of the long non-coding RNA AFAP1-AS1 affects the proliferation, invasion and survival of tongue squamous cell carcinoma via the Wnt/beta-catenin signaling pathway. Mol Cancer (2018) 17(1):3. doi: 10.1186/s12943-0170752-2

24. Tan JW, Xiang LX, Xu GC. LncRNA MEG3 suppresses migration and promotes apoptosis by sponging miR-548d-3p to modulate JAK-STAT pathway in oral squamous cell carcinoma. IUBMB Life (2019) 71(7):88290. doi: 10.1002/iub. 2012

25. Zhang H, Zhang N, Liu Y, Su P, Liang Y, Li Y, et al. Epigenetic Regulation of NAMPT by NAMPT-AS Drives Metastatic Progression in Triple-Negative Breast Cancer. Cancer Res (2019) 79(13):3347-59. doi: 10.1158/00085472.Can-18-3418

26. Liu M, Zhong J, Zeng Z, Huang K, Ye Z, Deng S, et al. Hypoxia-induced feedback of HIF-1 $\alpha$ and lncRNA-CF129 contributes to pancreatic cancer progression through stabilization of p53 protein. Theranostics (2019) 9 (16):4795-810. doi: 10.7150/thno.30988

27. Yamamura S, Imai-Sumida M, Tanaka Y, Dahiya R. Interaction and crosstalk between non-coding RNAs. Cell Mol Life Sci (2018) 75(3):467-84. doi: 10.1007/s00018-017-2626-6

28. Liu Z, Wang Y, Wang L, Yao B, Sun L, Liu R, et al. Long non-coding RNA AGAP2-AS1, functioning as a competitive endogenous RNA, upregulates ANXA11 expression by sponging miR-16-5p and promotes proliferation and metastasis in hepatocellular carcinoma. J Exp Clin Cancer Res (2019) 38 (1):194. doi: 10.1186/s13046-019-1188-x

29. Pan J, Fang S, Tian H, Zhou C, Zhao X, Tian H, et al. IncRNA JPX/miR-33a$5 \mathrm{p} /$ Twist 1 axis regulates tumorigenesis and metastasis of lung cancer by activating Wnt/ $\beta$-catenin signaling. Mol Cancer (2020) 19(1):9. doi: 10.1186/ s12943-020-1133-9

30. Welch DR, Hurst DR. Defining the Hallmarks of Metastasis. Cancer Res (2019) 79(12):3011-27. doi: 10.1158/0008-5472.Can-19-0458

31. Kou N, Liu S, Li XJ, Li WW, Zhong WJ, Gui L, et al. H19 Facilitates Tongue Squamous Cell Carcinoma Migration and Invasion via Sponging miR-let-7. Oncol Res (2019) 27(2):173-82. doi: 10.3727/096504018x15202945197589

32. Hong Y, He H, Sui W, Zhang J, Zhang S, Yang D. [Corrigendum] Long non -coding RNA H19 promotes cell proliferation and invasion by acting as a ceRNA of miR-138 and releasing EZH2 in oral squamous cell carcinoma. Int J Oncol (2018) 53(2):915. doi: 10.3892/ijo.2018.4428

33. Li MH, Ning J, Li ZH, Wang J, Zhao CO, Wang LE. LINC00152 promotes the growth and invasion of oral squamous cell carcinoma by regulating miR139-5p. Onco Targets Ther (2018) 11:6295-304. doi: 10.2147/ott.S168807

34. Li XH, Rui B, Cao YB, Gong XJ, Li HJ. Long non-coding RNA LINC00152 acts as a sponge of miRNA-193b-3p to promote tongue squamous cell carcinoma progression. Oncol Lett (2020) 19(3):2035-42. doi: 10.3892/ ol.2020.11293

35. Niu XY, Yang B, Liu F, Fang QG. LncRNA HOXA11-AS promotes OSCC progression by sponging miR-98-5p to upregulate YBX2 expression. BioMed Pharmacother (2020) 121:10. doi: 10.1016/j.biopha.2019.109623

36. Zeng BH, Li Y, Jiang FF, Wei CB, Chen GH, Zhang WL, et al. LncRNA GAS5 suppresses proliferation, migration, invasion, and epithelial-mesenchymal transition in oral squamous cell carcinoma by regulating the miR-21/PTEN axis. Exp Cell Res (2019) 374(2):365-73. doi: 10.1016/j.yexcr.2018.12.014

37. Zhang T-H, Liang L-Z, Liu X-L, Wu J-N, Su K, Chen J-Y, et al. Long noncoding RNA MALAT1 interacts with miR-124 and modulates tongue cancer growth by targeting JAG1. Oncol Rep (2017) 37(4):2087-94. doi: 10.3892/ or.2017.5445

38. Yuan J, Xu XJ, Lin Y, Chen QY, Sun WJ, Tang L, et al. LncRNA MALAT1 expression inhibition suppresses tongue squamous cell carcinoma proliferation, migration and invasion by inactivating PI3K/Akt pathway and downregulating MMP-9 expression. Eur Rev Med Pharmacol Sci (2019) 23(1):198-206. doi: 10.26355/eurrev_201901_16765

39. Liang J, Liang LZ, Ouyang KX, Li ZQ, Yi XP. MALATI induces tongue cancer cells' EMT and inhibits apoptosis through Wnt/beta-catenin signaling pathway. J Oral Pathol Med (2017) 46(2):98-105. doi: 10.1111/ jop. 12466

40. Zhou X, Liu S, Cai G, Kong L, Zhang T, Ren Y, et al. Long Non Coding RNA MALAT1 Promotes Tumor Growth and Metastasis by inducing EpithelialMesenchymal Transition in Oral Squamous Cell Carcinoma. Sci Rep (2015) 5:15972. doi: 10.1038/srep 15972

41. Lu MY, Liao YW, Chen PY, Hsieh PL, Fang CY, Wu CY, et al. Targeting LncRNA HOTAIR suppresses cancer stemness and metastasis in oral carcinomas stem cells through modulation of EMT. Oncotarget (2017) 8 (58):98542-52. doi: 10.18632/oncotarget.21614

42. Wu YS, Zhang L, Zhang L, Wang Y, Li H, Ren XB, et al. Long non-coding RNA HOTAIR promotes tumor cell invasion and metastasis by recruiting $\mathrm{EZH} 2$ and repressing E-cadherin in oral squamous cell carcinoma. Int $J$ Oncol (2015) 46(6):2586-94. doi: 10.3892/ijo.2015.2976

43. Tao DT, Zhang ZX, Liu X, Zhang ZW, Fu Y, Zhang P, et al. LncRNA HOTAIR promotes the invasion and metastasis of oral squamous cell carcinoma through metastasis-associated gene 2. Mol Carcinog (2020) 59 (4):353-64. doi: 10.1002/mc.23159

44. Su SC, Hsieh MJ, Lin CW, Chuang CY, Liu YF, Yeh CM, et al. Impact of HOTAIR Gene Polymorphism and Environmental Risk on Oral Cancer. J Dental Res (2018) 97(6):717-24. doi: 10.1177/0022034517749451

45. Liu Z, Wu C, Xie N, Wang P. Long non-coding RNA MEG3 inhibits the proliferation and metastasis of oral squamous cell carcinoma by regulating the WNT/beta-catenin signaling pathway. Oncol Lett (2017) 14(4):4053-8. doi: 10.3892/ol.2017.6682

46. Zhang LL, Hu D, Zou LH. Low expression of lncRNA MEG3 promotes the progression of oral squamous cell carcinoma by targeting miR-21. Eur Rev Med Pharmacol Sci (2018) 22(23):8315-23. doi: 10.26355/eurrev_201812_16529

47. Yang YT, Wang YF, Lai JY, Shen SY, Wang F, Kong J, et al. Long non-coding RNA UCA1 contributes to the progression of oral squamous cell carcinoma by regulating the WNT/beta-catenin signaling pathway. Cancer Sci (2016) 107(11):1581-9. doi: 10.1111/cas.13058 
48. Duan Q, Xu M, Wu M, Zhang X, Gan M, Jiang H. Long noncoding RNA UCA1 promotes cell growth, migration, and invasion by targeting miR-1433p in oral squamous cell carcinoma. Cancer Med (2020) 9(9):3115-29. doi: $10.1002 / \mathrm{cam} 4.2808$

49. Liang S, Zhang S, Wang P, Yang C, Shang C, Yang J, et al. LncRNA, TUG1 regulates the oral squamous cell carcinoma progression possibly via interacting with Wnt/beta-catenin signaling. Gene (2017) 608:49-57. doi: 10.1016/j.gene.2017.01.024

50. Liu SY, Liu LH, Hu WW, Wang M. Long noncoding RNA TUG1 regulates the development of oral squamous cell carcinoma through sponging miR524-5p to mediate DLX1 expression as a competitive endogenous RNA. J Cell Physiol (2019) 234(11):20206-16. doi: 10.1002/jcp.28620

51. Yan G, Wang X, Yang M, Lu L, Zhou Q. Long non-coding RNA TUG1 promotes progression of oral squamous cell carcinoma through upregulating FMNL2 by sponging miR-219. Am J Cancer Res (2017) 7(9):1899-912.

52. Zuo ZB, Ma L, Gong ZD, Xue LD, Wang QB. Long non-coding RNA CASC15 promotes tongue squamous carcinoma progression through targeting miR-33a-5p. Environ Sci Pollut Res (2018) 25(22):22205-12. doi: 10.1007/s11356-018-2300-Z

53. Wang Y, Zhang XJ, Wang Z, Hu QC, Wu J, Li YY, et al. LncRNA-p23154 promotes the invasion-metastasis potential of oral squamous cell carcinoma by regulating Glut1-mediated glycolysis. Cancer Lett (2018) 434:172-83. doi: $10.1016 /$ j.canlet.2018.07.016

54. Li GH, Ma ZH, Wang X. Long non-coding RNA CCAT1 is a prognostic biomarker for the progression of oral squamous cell carcinoma via miR181a-mediated Wnt/beta-catenin signaling pathway. Cell Cycle (2019) 18 (21):2902-13. doi: 10.1080/15384101.2019.1662257

55. Sun MY, Shen ZY. Knockdown of Long Non-Coding RNA (lncRNA) Colon Cancer-Associated Transcript-1 (CCAT1) Suppresses Oral Squamous Cell Carcinoma Proliferation, Invasion, and Migration by Inhibiting the Discoidin Domain Receptor 2 (DDR2)/ERK/AKT Axis. Med Sci Monit (2020) 26:10. doi: 10.12659/msm.920020

56. Chen ZF, Tao Q, Qiao B, Zhang LT. Silencing of LINC01116 suppresses the development of oral squamous cell carcinoma by up-regulating microRNA136 to inhibit FN1. Cancer Manage Res (2019) 11:6043-59. doi: 10.2147/ cmar.S197583

57. Liu X, Zhang B, Jia Y, Fu M. SNHG17 enhances the malignant characteristics of tongue squamous cell carcinoma by acting as a competing endogenous RNA on microRNA-876 and thereby increasing specificity protein 1 expression. Cell Cycle (2020) 19(6):711-25. doi: 10.1080/15384101.2020.1727399

58. Li S, Zhang S, Chen J. c-Myc induced upregulation of long non-coding RNA SNHG16 enhances progression and carcinogenesis in oral squamous cell carcinoma. Cancer Gene Ther (2019) 26(11-12):400-10. doi: 10.1038/ s41417-018-0072-8

59. Zhao Y, Wang J, Ma K. [Knockdown of IncRNA SNHG6 inhibites the proliferation and epithelial mesenchymal transition in tongue cancer cells]. Xi Bao Yu Fen Zi Mian Yi Xue Za Zhi (2018) 34(9):806-11. doi: 10.13423/ j.cnki.cjcmi.008671

60. Liu Z, Tao H. Small nucleolar RNA host gene 3 facilitates cell proliferation and migration in oral squamous cell carcinoma via targeting nuclear transcription factor Y subunit gamma. J Cell Biochem (2020) 121(3):21508. doi: $10.1002 / \mathrm{jcb} .29421$

61. Li X, Ma C, Zhang L, Li N, Zhang X, He J, et al. LncRNAAC132217.4, a KLF8-regulated long non-coding RNA, facilitates oral squamous cell carcinoma metastasis by upregulating IGF2 expression. Cancer Lett (2017) 407:45-56. doi: 10.1016/j.canlet.2017.08.007

62. Jiang YY, Cao W, Wu K, Qin X, Wang XN, Li Y, et al. LncRNA LINC00460 promotes EMT in head and neck squamous cell carcinoma by facilitating peroxiredoxin-1 into the nucleus. J Exp Clin Cancer Res (2019) 38(1):19. doi: 10.1186/s13046-019-1364-Z

63. Liu Z, Li H, Fan SM, Lin H, Lian WW. STAT3-induced upregulation of long noncoding RNA HNF1A-AS1 promotes the progression of oral squamous cell carcinoma via activating Notch signaling pathway. Cancer Biol Ther (2019) 20(4):444-53. doi: 10.1080/15384047.2018.1529119

64. Liu M, Gao X, Liu CL. Increased expression of IncRNA FTH1P3 promotes oral squamous cell carcinoma cells migration and invasion by enhancing PI3K/Akt/GSK3b/Wnt/beta-catenin signaling. Eur Rev Med Pharmacol Sci (2018) 22(23):8306-14. doi: 10.26355/eurrev_201812_16528
65. Shih JW, Chiang WF, Wu ATH, Wu MH, Wang LY, Yu YL, et al. Long noncoding RNA LncHIFCAR/MIR31HG is a HIF-1alpha co-activator driving oral cancer progression. Nat Commun (2017) 8:15874. doi: $10.1038 /$ ncomms 15874

66. Zhu G, Wang S, Chen J, Wang Z, Liang X, Wang X, et al. Long noncoding RNA HAS2-AS1 mediates hypoxia-induced invasiveness of oral squamous cell carcinoma. Mol Carcinog (2017) 56(10):2210-22. doi: 10.1002/ mc. 22674

67. Mu MK, Li Y, Zhan YB, Li X, Zhang B. Knockdown of HOXA transcript at the distal tip suppresses the growth and invasion and induces apoptosis of oral tongue squamous carcinoma cells. Onco Targets Ther (2018) 11:803344. doi: $10.2147 /$ ott.S174637

68. Xiong L, Tang Y, Tang J, Liu Z, Wang X. Downregulation of IncRNA HOTTIP Suppresses the Proliferation, Migration, and Invasion of Oral Tongue Squamous Cell Carcinoma by Regulation of HMGA2-Mediated Wnt/B-Catenin Pathway. Cancer Biother Radiopharm (2020) 35(9):720-30. doi: $10.1089 / \mathrm{cbr} .2019 .3017$

69. Zhang CQ, Bao CC, Zhang XX, Lin XS, Pan D, Chen YZ. Knockdown of lncRNA LEF1-AS1 inhibited the progression of oral squamous cell carcinoma (OSCC) via Hippo signaling pathway. Cancer Biol Ther (2019) 20(9):1213-22. doi: 10.1080/15384047.2019.1599671

70. Zhou NG, Liao W, Huang ZK, Hu ZW, Huang WZ, Wang QT. Overexpression of long non-coding RNA CCAT2 predicts a poor prognosis in patients with oral squamous cell carcinoma. Int J Clin Exp Pathol (2016) 9(1):110-7.

71. Huang Z, Sang T, Zheng Y, Wu J. Long non-coding RNA PANDAR overexpression serves as a poor prognostic biomarker in oral squamous cell carcinoma. Int J Clin Exp Pathol (2018) 11(5):2728-34.

72. Hu D, Zhong T, Dai Q. Long Non-Coding RNA NKILA Reduces Oral Squamous Cell Carcinoma Development Through the NF-KappaB Signaling Pathway. Technol Cancer Res Treat (2020) 19:1533033820960747. doi: 10.1177/1533033820960747

73. Wu K, Jiang Y, Zhou W, Zhang B, Li Y, Xie F, et al. Long Noncoding RNA RC3H2 Facilitates Cell Proliferation and Invasion by Targeting MicroRNA101-3p/EZH2 Axis in OSCC. Mol Ther Nucleic Acids (2020) 20:97-110. doi: 10.1016/j.omtn.2020.02.006

74. Jin N, Jin NQ, Bu WH, Li X, Liu LL, Wang ZL, et al. Long non-coding RNA TIRY promotes tumor metastasis by enhancing epithelial-to-mesenchymal transition in oral cancer. Exp Biol Med (2020) 245(7):585-96. doi: 10.1177/ 1535370220903673

75. Lee JM, Dedhar S, Kalluri R, Thompson EW. The epithelial-mesenchymal transition: new insights in signaling, development, and disease. J Cell Biol (2006) 172(7):973-81. doi: 10.1083/jcb.200601018

76. Xia E, Shen Y, Bhandari A, Zhou X, Wang Y, Yang F, et al. Long non-coding RNA LINC00673 promotes breast cancer proliferation and metastasis through regulating $\mathrm{B} 7-\mathrm{H} 6$ and epithelial-mesenchymal transition. Am J Cancer Res (2018) 8(7):1273-87.

77. Velmurugan BK, Yeh KT, Hsieh MJ, Yeh CM, Lin CC, Kao CY, et al. UNC13C Suppress Tumor Progression via Inhibiting EMT Pathway and Improves Survival in Oral Squamous Cell Carcinoma. Front Oncol (2019) 9:728:728. doi: 10.3389/fonc.2019.00728

78. Zhang Q, Li Y, Zhao R, Wang X, Fan C, Xu Y, et al. The gain-of-function mutation E76K in SHP2 promotes CAC tumorigenesis and induces EMT via the Wnt/beta-catenin signaling pathway. Mol Carcinog (2018) 57(5):619-28. doi: $10.1002 / \mathrm{mc} .22785$

79. Yi Y, Wang Z, Sun Y, Chen J, Zhang B, Wu M, et al. The EMT-related transcription factor snail up-regulates FAPalpha in malignant melanoma cells. Exp Cell Res (2018) 364(2):160-7. doi: 10.1016/j.yexcr.2018.01.039

80. Xue M, Chen LY, Wang WJ, Su TT, Shi LH, Wang L, et al. HOTAIR induces the ubiquitination of Runx 3 by interacting with Mex3b and enhances the invasion of gastric cancer cells. Gastric Cancer (2018) 21(5):756-64. doi: 10.1007/s10120-018-0801-6

81. Li Y, Chen F, Shen W, Li B, Xiang R, Qu L, et al. WDR74 induces nuclear $\beta$ catenin accumulation and activates Wnt-responsive genes to promote lung cancer growth and metastasis. Cancer Lett (2020) 471:103-15. doi: 10.1016/ j.canlet.2019.12.011

82. He Y, Davies CM, Harrington BS, Hellmers L, Sheng Y, Broomfield A, et al. CDCP1 enhances Wnt signaling in colorectal cancer promoting nuclear 
localization of $\beta$-catenin and E-cadherin. Oncogene (2020) 39(1):219-33. doi: 10.1038/s41388-019-0983-3

83. Eyre R, Alférez DG, Santiago-Gómez A, Spence K, McConnell JC, Hart C, et al. Microenvironmental IL1 $\beta$ promotes breast cancer metastatic colonisation in the bone via activation of Wnt signalling. Nat Commun (2019) 10(1):5016. doi: 10.1038/s41467-019-12807-0

84. Li BQ, Liang ZY, Seery S, Liu QF, You L, Zhang TP, et al. WT1 associated protein promotes metastasis and chemo-resistance to gemcitabine by stabilizing Fak mRNA in pancreatic cancer. Cancer Lett (2019) 451:48-57. doi: 10.1016/j.canlet.2019.02.043

85. Zhang X, Wang S, Wang H, Cao J, Huang X, Chen Z, et al. Circular RNA circNRIP1 acts as a microRNA-149-5p sponge to promote gastric cancer progression via the AKT1/mTOR pathway. Mol Cancer (2019) 18(1):20. doi: 10.1186/s12943-018-0935-5

86. Huang K, Liang Q, Zhou Y, Jiang LL, Gu WM, Luo MY, et al. A Novel Allosteric Inhibitor of Phosphoglycerate Mutase 1 Suppresses Growth and Metastasis of Non-Small-Cell Lung Cancer. Cell Metab (2019) 30(6):110719.e8. doi: 10.1016/j.cmet.2019.09.014

87. Dimitrakopoulos FD, Kottorou AE, Kalofonou M, Kalofonos HP. The Fire Within: NF- $\mathrm{KB}$ Involvement in Non-Small Cell Lung Cancer. Cancer Res (2020) 80(19):4025-36. doi: 10.1158/0008-5472.Can-19-3578

88. Khan H, Ullah H, Castilho P, Gomila AS, D’Onofrio G, Filosa R, et al. Targeting $\mathrm{NF}-\mathrm{KB}$ signaling pathway in cancer by dietary polyphenols. Crit Rev Food Sci Nutr (2020) 60(16):2790-800. doi: 10.1080/10408398.2019.1661827

89. Zheng J, Zhang H, Ma R, Liu H, Gao P. Long non-coding RNA KRT19P3 suppresses proliferation and metastasis through COPS7A-mediated NF- $\mathrm{KB}$ pathway in gastric cancer. Oncogene (2019) 38(45):7073-88. doi: 10.1038/ s41388-019-0934-Z

90. Su K, Zhao Q, Bian A, Wang C, Cai Y, Zhang Y. A novel positive feedback regulation between long noncoding RNA UICC and IL-6/STAT3 signaling promotes cervical cancer progression. Am J Cancer Res (2018) 8(7):1176-89.

91. Alves A, Diel L, Ramos G, Pinto A, Bernardi L, Yates J, et al. Tumor microenvironment and Oral Squamous Cell Carcinoma: A crosstalk between the inflammatory state and tumor cell migration. Oral Oncol (2020) 112:105038. doi: 10.1016/j.oraloncology.2020.105038

92. Qu Y, He Y, Yang Y, Li S, An W, Li Z, et al. ALDH3A1 acts as a prognostic biomarker and inhibits the epithelial mesenchymal transition of oral squamous cell carcinoma through IL-6/STAT3 signaling pathway. J Cancer (2020) 11(9):2621-31. doi: 10.7150/jca.40171

93. Tripathi V, Ellis JD, Shen Z, Song DY, Pan Q, Watt AT, et al. The nuclearretained noncoding RNA MALAT1 regulates alternative splicing by modulating SR splicing factor phosphorylation. Mol Cell (2010) 39 (6):925-38. doi: 10.1016/j.molcel.2010.08.011

94. Li GQ, Fang YX, Liu Y, Meng FR, Wu X, Zhang CW, et al. MALAT1-Driven Inhibition of Wnt Signal Impedes Proliferation and Inflammation in Fibroblast-Like Synoviocytes Through CTNNB1 Promoter Methylation in Rheumatoid Arthritis. Hum Gene Ther (2019) 30(8):1008-22. doi: 10.1089/ hum.2018.212

95. Lin N, Yao Z, Xu M, Chen J, Lu Y, Yuan L, et al. Long noncoding RNA MALAT1 potentiates growth and inhibits senescence by antagonizing ABI3BP in gallbladder cancer cells. J Exp Clin Cancer Res CR (2019) 38 (1):244. doi: 10.1186/s13046-019-1237-5

96. Jin D, Guo J, Wu Y, Du J, Yang L, Wang X, et al. m(6)A mRNA methylation initiated by METTL3 directly promotes YAP translation and increases YAP activity by regulating the MALAT1-miR-1914-3p-YAP axis to induce NSCLC drug resistance and metastasis. J Hematol Oncol (2019) 12(1):135. doi: 10.1186/s13045-019-0830-6

97. Yoshimura H, Matsuda Y, Yamamoto M, Michishita M, Takahashi K, Sasaki N, et al. Reduced expression of the H19 long non-coding RNA inhibits pancreatic cancer metastasis. Lab Invest J Tech Methods Pathol (2018) 98(6):814-24. doi: 10.1038/s41374-018-0048-1

98. Zhang Y, Zhu R, Wang J, Cui Z, Wang Y, Zhao Y. Upregulation of IncRNA H19 promotes nasopharyngeal carcinoma proliferation and metastasis in let-7 dependent manner. Artif Cells Nanomed Biotechnol (2019) 47(1):385461. doi: 10.1080/21691401.2019.1669618

99. Zhou Y, Zhang Y. Inhibition of LncRNAH19 has the effect of anti-tumour and enhancing sensitivity to Gefitinib and Chemotherapy in Non-small-cell lung cancer in vivo. J Cell Mol Med (2020) 24(10):5811-6. doi: 10.1111/ jcmm. 15245

100. Lv M, Zhong Z, Huang M, Tian Q, Jiang R, Chen J. IncRNA H19 regulates epithelial-mesenchymal transition and metastasis of bladder cancer by miR29b-3p as competing endogenous RNA. Biochim Biophys Acta Mol Cell Res (2017) 1864(10):1887-99. doi: 10.1016/j.bbamcr.2017.08.001

101. Zhou W, Ye XL, Xu J, Cao MG, Fang ZY, Li LY, et al. The lncRNA H19 mediates breast cancer cell plasticity during EMT and MET plasticity by differentially sponging miR-200b/c and let-7b. Sci Signal (2017) 10(483): eaak9557. doi: 10.1126/scisignal.aak9557

102. Zhu Y, Chen P, Gao Y, Ta N, Zhang Y, Cai J, et al. MEG3 Activated by Vitamin D Inhibits Colorectal Cancer Cells Proliferation and Migration via Regulating Clusterin. EBioMedicine (2018) 30:148-57. doi: 10.1016/ j.ebiom.2018.03.032

103. Huang C, Liao X, Jin H, Xie F, Zheng F, Li J, et al. MEG3, as a Competing Endogenous RNA, Binds with miR-27a to Promote PHLPP2 Protein Translation and Impairs Bladder Cancer Invasion. Mol Ther Nucleic Acids (2019) 16:51-62. doi: 10.1016/j.omtn.2019.01.014

104. Zhou H, Tang K, Liu H, Zeng J, Li H, Yan L, et al. Regulatory Network of Two Tumor-Suppressive Noncoding RNAs Interferes with the Growth and Metastasis of Renal Cell Carcinoma. Mol Ther Nucleic Acids (2019) 16:55465. doi: 10.1016/j.omtn.2019.04.005

105. Cai Q, Wang ZQ, Wang SH, Li C, Zhu ZG, Quan ZW, et al. Upregulation of long non-coding RNA LINC00152 by SP1 contributes to gallbladder cancer cell growth and tumor metastasis via PI3K/AKT pathway. Am J Trans Res (2016) 8(10):4068-81.

106. Ou C, Sun Z, He X, Li X, Fan S, Zheng X, et al. Targeting YAP1/LINC00152/ FSCN1 Signaling Axis Prevents the Progression of Colorectal Cancer. Adv Sci (Weinh) (2020) 7(3):1901380. doi: 10.1002/advs.201901380

107. Yue B, Liu C, Sun H, Liu M, Song C, Cui R, et al. A Positive Feed-Forward Loop between LncRNA-CYTOR and Wnt/B-Catenin Signaling Promotes Metastasis of Colon Cancer. Mol Ther (2018) 26(5):1287-98. doi: 10.1016/ j.ymthe.2018.02.024

108. Shi Y, Sun H. Down-regulation of lncRNA LINC00152 Suppresses Gastric Cancer Cell Migration and Invasion Through Inhibition of the ERK/MAPK Signaling Pathway. Onco Targets Ther (2020) 13:2115-24. doi: 10.2147/ ott.S217452

109. Yu JJ, Liu Y, Guo C, Zhang SS, Gong ZJ, Tang YY, et al. Upregulated long non-coding RNA LINC00152 expression is associated with progression and poor prognosis of tongue squamous cell carcinoma. J Cancer (2017) 8 (4):523-30. doi: $10.7150 /$ jca. 17510

110. Wang XS, Zhang Z, Wang HC, Cai JL, Xu QW, Li MQ, et al. Rapid identification of UCA1 as a very sensitive and specific unique marker for human bladder carcinoma. Clin Cancer Res (2006) 12(16):4851-8. doi: 10.1158/1078-0432.Ccr-06-0134

111. Xue M, Chen W, Xiang A, Wang R, Chen H, Pan J, et al. Hypoxic exosomes facilitate bladder tumor growth and development through transferring long non-coding RNA-UCA1. Mol Cancer (2017) 16(1):143. doi: 10.1186/s12943017-0714-8

112. Gong P, Qiao F, Wu H, Cui H, Li Y, Zheng Y, et al. LncRNA UCA1 promotes tumor metastasis by inducing miR-203/ZEB2 axis in gastric cancer. Cell Death Dis (2018) 9(12):1158. doi: 10.1038/s41419-018-1170-0

113. Su Y, Zhou Y, Sun YJ, Wang YL, Yin JY, Huang YJ, et al. Macrophagederived CCL18 promotes osteosarcoma proliferation and migration by upregulating the expression of UCA1. J Mol Med (Berlin Germany) (2019) 97(1):49-61. doi: 10.1007/s00109-018-1711-0

114. Li GY, Wang W, Sun JY, Xin B, Zhang X, Wang T, et al. Long non-coding RNAs AC026904.1 and UCA1: a "one-two punch" for TGF- $\beta$-induced SNAI2 activation and epithelial-mesenchymal transition in breast cancer. Theranostics (2018) 8(10):2846-61. doi: 10.7150/thno.23463

115. Fang Z, Wu L, Wang L, Yang Y, Meng Y, Yang H. Increased expression of the long non-coding RNA UCA1 in tongue squamous cell carcinomas: a possible correlation with cancer metastasis. Oral Surg Oral Med Oral Pathol Oral Radiol (2014) 117(1):89-95. doi: 10.1016/j.oooo.2013.09.007

116. Shen X, Hu X, Mao J, Wu Y, Liu H, Shen J, et al. The long noncoding RNA TUG1 is required for TGF- $\beta$ /TWIST1/EMT-mediated metastasis in colorectal cancer cells. Cell Death Dis (2020) 11(1):65. doi: 10.1038/ s41419-020-2254-1 
117. Yu X, Hu L, Li S, Shen J, Wang D, Xu R, et al. Long non-coding RNA Taurine upregulated gene 1 promotes osteosarcoma cell metastasis by mediating HIF-1 $\alpha$ via miR-143-5p. Cell Death Dis (2019) 10(4):280. doi: 10.1038/ s41419-019-1509-1

118. Lin YH, Wu MH, Huang YH, Yeh CT, Cheng ML, Chi HC, et al. Taurine upregulated gene 1 functions as a master regulator to coordinate glycolysis and metastasis in hepatocellular carcinoma. Hepatology (2018) 67(1):188-203. doi: 10.1002/hep.29462

119. Yang CZ, Ma J, Zhu DW, Liu Y, Montgomery B, Wang LZ, et al. GDF15 is a potential predictive biomarker for TPF induction chemotherapy and promotes tumorigenesis and progression in oral squamous cell carcinoma. Ann Oncol (2014) 25(6):1215-22. doi: 10.1093/annonc/mdu120

120. Zhang H, Zhao L, Wang YX, Xi M, Liu SL, Luo LL. Long non-coding RNA HOTTIP is correlated with progression and prognosis in tongue squamous cell carcinoma. Tumour Biol (2015) 36(11):8805-9. doi: 10.1007/s13277-015-3645-2

121. Wang FW, Cao CH, Han K, Zhao YX, Cai MY, Xiang ZC, et al. APCactivated long noncoding RNA inhibits colorectal carcinoma pathogenesis through reduction of exosome production. J Clin Invest (2019) 129(2):72743. doi: $10.1172 /$ jci122478

122. Deng SJ, Chen HY, Ye Z, Deng SC, Zhu S, Zeng Z, et al. Hypoxia-induced LncRNA-BX111 promotes metastasis and progression of pancreatic cancer through regulating ZEB1 transcription. Oncogene (2018) 37(44):5811-28. doi: 10.1038/s41388-018-0382-1

123. Snellenberg S, Cillessen SA, Van Criekinge W, Bosch L, Meijer CJ, Snijders $\mathrm{PJ}$, et al. Methylation-mediated repression of PRDM14 contributes to apoptosis evasion in HPV-positive cancers. Carcinogenesis (2014) 35 (11):2611-8. doi: 10.1093/carcin/bgu197

124. Wu T, Dai Y. Tumor microenvironment and therapeutic response. Cancer Lett (2017) 387:61-8. doi: 10.1016/j.canlet.2016.01.043

125. Jackstadt R, van Hooff SR, Leach JD, Cortes-Lavaud X, Lohuis JO, Ridgway RA, et al. Epithelial NOTCH Signaling Rewires the Tumor Microenvironment of Colorectal Cancer to Drive Poor-Prognosis Subtypes and Metastasis. Cancer Cell (2019) 36(3):319-36. doi: 10.1016/j.ccell.2019.08.003

126. Zanconato F, Cordenonsi M, Piccolo S. YAP and TAZ: a signalling hub of the tumour microenvironment. Nat Rev Cancer (2019) 19(8):454-64. doi: 10.1038/s41568-019-0168-y

127. Jiang E, Xu Z, Wang M, Yan T, Huang C, Zhou X, et al. Tumoral microvesicle-activated glycometabolic reprogramming in fibroblasts promotes the progression of oral squamous cell carcinoma. FASEB J (2019) 33(4):5690-703. doi: 10.1096/fj.201802226R

128. Kong J, Tian H, Zhang F, Zhang Z, Li J, Liu X, et al. Extracellular vesicles of carcinoma-associated fibroblasts creates a pre-metastatic niche in the lung through activating fibroblasts. Mol Cancer (2019) 18(1):175. doi: 10.1186/ s12943-019-1101-4

129. Ren Y, Jia HH, Xu YQ, Zhou X, Zhao XH, Wang YF, et al. Paracrine and epigenetic control of CAF-induced metastasis: the role of HOTAIR stimulated by TGF-ß1 secretion. Mol Cancer (2018) 17(1):5. doi: 10.1186/ s12943-018-0758-4

130. Zhao L, Ji G, Le X, Wang C, Xu L, Feng M, et al. Long Noncoding RNA LINC00092 Acts in Cancer-Associated Fibroblasts to Drive Glycolysis and Progression of Ovarian Cancer. Cancer Res (2017) 77(6):1369-82. doi: 10.1158/0008-5472.Can-16-1615

131. Ren J, Ding L, Zhang D, Shi G, Xu Q, Shen S, et al. Carcinoma-associated fibroblasts promote the stemness and chemoresistance of colorectal cancer by transferring exosomal lncRNA H19. Theranostics (2018) 8(14):3932-48. doi: $10.7150 /$ thno. 25541

132. Cen B, Lang JD, Du Y, Wei J, Xiong Y, Bradley N, et al. Prostaglandin E(2) Induces miR675-5p to Promote Colorectal Tumor Metastasis via Modulation of p53 Expression. Gastroenterology (2020) 158(4):971-84. doi: 10.1053/j.gastro.2019.11.013

133. Chen C, Luo Y, He W, Zhao Y, Kong Y, Liu H, et al. Exosomal long noncoding RNA LNMAT2 promotes lymphatic metastasis in bladder cancer. J Clin Invest (2020) 130(1):404-21. doi: 10.1172/jci130892

134. Liang ZX, Liu HS, Wang FW, Xiong L, Zhou C, Hu T, et al. LncRNA RPPH1 promotes colorectal cancer metastasis by interacting with TUBB3 and by promoting exosomes-mediated macrophage M2 polarization. Cell Death Dis (2019) 10(11):829. doi: 10.1038/s41419-019-2077-0

135. Chen CW, Fu M, Du ZH, Zhao F, Yang WW, Xu LH, et al. Long Noncoding RNA MRPL23-AS1 Promoteoid Cystic Carcinoma Lung Metastasis. Cancer Res (2020) 88(11):2273-85. doi: 10.1158/00085472.Can-19-0819

136. O’Brien K, Breyne K, Ughetto S, Laurent LC, Breakefield XO. RNA delivery by extracellular vesicles in mammalian cells and its applications. Nat Rev Mol Cell Biol (2020) 22(10):585-606. doi: 10.1038/s41580-020-0251-y

137. Li X, Lv X, Li Z, Li C, Li X, Xiao J, et al. Long Noncoding RNA ASLNC07322 Functions in VEGF-C Expression Regulated by Smad4 during Colon Cancer Metastasis. Mol Ther Nucleic Acids (2019) 18:851-62. doi: 10.1016/ j.omtn.2019.10.012

138. Huang YL, Liang CY, Ritz D, Coelho R, Septiadi D, Estermann M, et al. Collagen-rich omentum is a premetastatic niche for integrin $\alpha 2$-mediated peritoneal metastasis. Elife (2020) 9:e59442. doi: 10.7554/eLife.59442

Conflict of Interest: The authors declare that the research was conducted in the absence of any commercial or financial relationships that could be construed as a potential conflict of interest.

Copyright (c) $2021 \mathrm{Xu}$, Jiang, Shao and Shang. This is an open-access article distributed under the terms of the Creative Commons Attribution License (CC BY). The use, distribution or reproduction in other forums is permitted, provided the original author(s) and the copyright owner(s) are credited and that the original publication in this journal is cited, in accordance with accepted academic practice. No use, distribution or reproduction is permitted which does not comply with these terms. 